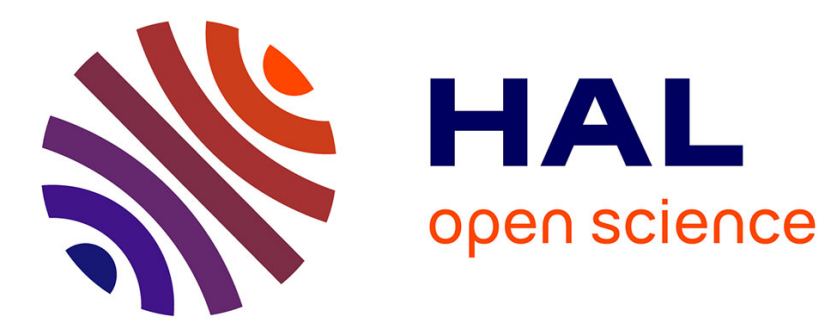

\title{
Hybrid numerical model for acoustic propagation through sheared flows
}

Karim Hamiche, Sophie Le Bras, Gwenael Gabard, Hadrien Beriot

\section{To cite this version:}

Karim Hamiche, Sophie Le Bras, Gwenael Gabard, Hadrien Beriot. Hybrid numerical model for acoustic propagation through sheared flows. Journal of Sound and Vibration, 2019, 463, pp.114951. 10.1016/j.jsv.2019.114951 . hal-02457796

\section{HAL Id: hal-02457796}

https://hal-univ-lemans.archives-ouvertes.fr/hal-02457796

Submitted on 21 Dec 2021

HAL is a multi-disciplinary open access archive for the deposit and dissemination of scientific research documents, whether they are published or not. The documents may come from teaching and research institutions in France or abroad, or from public or private research centers.
L'archive ouverte pluridisciplinaire HAL, est destinée au dépôt et à la diffusion de documents scientifiques de niveau recherche, publiés ou non, émanant des établissements d'enseignement et de recherche français ou étrangers, des laboratoires publics ou privés.

\section{(ㄷ)(1) $\$$}

Distributed under a Creative Commons Attribution - NonCommercial| 4.0 International 


\title{
Hybrid numerical model for acoustic propagation through sheared flows
}

\author{
Karim Hamiche ${ }^{\mathrm{a}}$, Sophie Le Bras ${ }^{\mathrm{a}}$, Gwénaël Gabard ${ }^{\mathrm{b}}$, Hadrien Bériot ${ }^{\mathrm{a}, *}$ \\ ${ }^{a}$ Siemens Industry Software N.V., Interleuvenlaan 68, Researchpark Z1, Leuven, Belgium \\ ${ }^{b}$ LAUM UMR CNRS 6613, Le Mans Université, Avenue Olivier Messiaen, 72085 Le Mans, France
}

\begin{abstract}
Simulating the propagation of sound in non-uniform flows remains challenging, especially for large, three-dimensional problems. To account for the sound refraction due to gradients of velocity and temperature, one has to solve the Linearised Euler Equations (LEE) which can be computationally expensive in three dimensions. Alternatively, one can use the Linearised Potential Equation (LPE) which is much cheaper but is limited to potential, isentropic flows. In this paper, a hybrid model combining the LEE and the LPE is proposed in order to simulate the sound propagation in sheared flows at a reasonable computational cost. The LEE are applied only in regions with strong sheared mean flows, the LPE is used everywhere else. The coupling between the LEE and the LPE consists in imposing relations for the characteristic waves propagating at the interface between the LEE and the LPE regions. In this study, the hybrid model is implemented in a high-order finite element solver in the frequency domain. Its performance is first assessed by simulating the propagation of planes waves in a uniform mean flow and the acoustic radiation from a semi-infinite duct in a strongly sheared non-isothermal jet flow. No significant spurious noise is produced at the LEELPE interfaces. The applicability of the hybrid model to industrial problems is then demonstrated by simulating the propagation of fan noise through the jet flow exiting from a turbofan exhaust. The use of the proposed hybrid model does not affect the propagation of the sound field while reducing the memory footprint by more than one order of magnitude compared to a full three-dimensional LEE simulation.
\end{abstract}

Keywords: $p$-FEM, linearised Euler equations, linearised potential equation, aeroacoustics

\section{Introduction}

Simulating the sound propagation in a non-uniform flow is important to predict noise emissions from various engineering systems, such as turbofan engines or ventilation systems. A number of propagation models are available for this purpose [1]. Amongst them, the linearised Euler equations (LEE) support the acoustic, hydrodynamic and 5 entropy waves, as well as their linear interactions. They also provide a full description of the refraction of acoustic waves as they propagate through a sheared mean flow. The LEE have been solved extensively both in the time domain $[2,3]$ and the frequency domain $[4,5,6]$. A downside of this model, when solved in the time domain, is the presence of hydrodynamic instabilities, which can be difficult to handle since they can dominate the acoustic field. Another major drawback is the computational cost associated to the resolution of the LEE. When including all three types of 10 waves, one has to solve for the perturbations of density, momentum and pressure. In the frequency domain, this results in very large linear systems of equations that require massive amounts of memory to solve using modern direct solvers such as MUMPS or PARDISO (iterative solvers are, as yet, not sufficiently robust for this type of applications).

The Linearised Potential Equation (LPE) provides a simplified model to describe the sound propagation in a mean flow. It is based on the assumption that vorticity and entropy effects are absent from both the base flow and the linear 15 perturbations. In this case one can write a differential equation for the acoustic velocity potential. Being a scalar equation, the LPE is much less costly to solve than the LEE. The LPE forms the basis for several commercial codes

*Correspondence to: Gwénaël Gabard, Le Mans Université, Avenue Olivier Messiaen, 72085 Le Mans, France 
that are used routinely in industry. However, the main limitation of this model is that it does not account for the refraction of sound through a sheared mean flow.

In this paper, a hybrid model which combines the LEE and LPE is proposed in order to address their respective shortcomings. The rationale is to use the LEE only in regions with strong shear mean flows, and to use the LPE everywhere else. With this hybrid approach, the computational cost is significantly reduced compared to a full LEE model and it provides a way to circumvent the inherent limitation of the LPE. The key to formulate such a hybrid model is the derivation of coupling conditions at the interface between the LEE and LPE regions. These conditions are obtained by writing characteristic wave relations through the LEE-LPE interface.

Several computational methods have been developed specifically to solve the LEE or the LPE, either in the time domain or in the frequency domain [7]. The present work will focus on frequency-domain simulations and use a high-order finite element method that has been developed both for the LPE [8, 9] and the LEE [6].

This paper is organized as follows. The next section presents the LEE and LPE, together with the associated variational formulations. Section 3 is concerned with the hybrid model and describes how the LEE and LPE models are coupled. The high-order finite element method used to solve both the LPE and the LEE is presented in Section 4. The performance of the hybrid numerical model is verified in Section 5 by simulating the propagation of a set of acoustic plane waves in a uniform mean flow, and the sound scattering from a semi-infinite duct in a strongly sheared non-isothermal flow. The applicability of the hybrid model to a turbofan exhaust is demonstrated in Section 6. Concluding remarks and perspectives of this work are given in Section 7.

\section{Governing equations}

\subsection{Linearised Euler Equations (LEE)}

We consider a perfect gas with isentropic disturbances (no viscous effect nor heat transfer). The density $\rho^{\prime}$, the velocity vector $\boldsymbol{u}^{\prime}=\left(u^{\prime}, v^{\prime}, w^{\prime}\right)$, and the pressure $p^{\prime}$ represent small perturbations around a steady mean flow of density $\rho_{0}$, velocity $\boldsymbol{u}_{0}=\left(u_{0}, v_{0}, w_{0}\right)$ and pressure $p_{0}$. The behavior of these perturbations is governed by the linearised Euler equations. In three-dimensional Cartesian coordinates, these equations can be written in conservative form as:

$$
\frac{\partial \boldsymbol{q}}{\partial t}+\frac{\partial \boldsymbol{A} \boldsymbol{q}}{\partial x}+\frac{\partial \boldsymbol{B} \boldsymbol{q}}{\partial y}+\frac{\partial \boldsymbol{C} \boldsymbol{q}}{\partial z}=\mathbf{0}
$$

where $\boldsymbol{q}=\left[\rho^{\prime},(\rho u)^{\prime},(\rho v)^{\prime},(\rho w)^{\prime}, p_{\mathrm{c}}^{\prime}\right]^{T}$ is the unknown vector, $p_{\mathrm{c}}=\left(p / p_{\infty}\right)^{1 / \gamma}$ is the non-dimensional pressure defined by Goldstein [10], $p_{\infty}$ is a reference pressure, $\gamma$ is the specific heat ratio, and.$^{T}$ is the transpose operator. The flux matrices $\boldsymbol{A}, \boldsymbol{B}$ and $\boldsymbol{C}$, defined from the steady mean flow properties, are given in Appendix A. These equations are solved in the frequency domain, assuming an implicit time dependence for the solution vector $\boldsymbol{q}(\boldsymbol{x}, t)=\boldsymbol{q}(\boldsymbol{x}, \omega) \exp (\mathrm{i} \omega t)$ where $\omega$ is the angular frequency. The LEE become:

$$
\mathrm{i} \omega \boldsymbol{q}+\frac{\partial \boldsymbol{A} \boldsymbol{q}}{\partial x}+\frac{\partial \boldsymbol{B} \boldsymbol{q}}{\partial y}+\frac{\partial \boldsymbol{C} \boldsymbol{q}}{\partial z}=\mathbf{0}
$$

For a domain $\Omega_{\mathrm{LEE}}$ delimited by the boundary $\Gamma_{\mathrm{LEE}}$, the variational formulation is defined as:

$$
\int_{\Omega_{\mathrm{LEE}}} \boldsymbol{\psi}^{\dagger}\left(\mathrm{i} \omega \boldsymbol{q}+\frac{\partial \boldsymbol{A} \boldsymbol{q}}{\partial x}+\frac{\partial \boldsymbol{B} \boldsymbol{q}}{\partial y}+\frac{\partial \boldsymbol{C} \boldsymbol{q}}{\partial z}\right) \mathrm{d} \Omega=0
$$

where $\psi$ is the test function vector and $\cdot^{\dagger}$ denotes the Hermitian transpose. Integrating by parts the flux terms leads to:

$$
\int_{\Omega_{\mathrm{LEE}}}\left(\mathrm{i} \omega \boldsymbol{\psi}^{\dagger} \boldsymbol{q}-\frac{\partial \boldsymbol{\psi}^{\dagger}}{\partial x} \boldsymbol{A} \boldsymbol{q}-\frac{\partial \boldsymbol{\psi}^{\dagger}}{\partial y} \boldsymbol{B} \boldsymbol{q}-\frac{\partial \boldsymbol{\psi}^{\dagger}}{\partial z} \boldsymbol{C} \boldsymbol{q}\right) \mathrm{d} \Omega+\int_{\Gamma_{\mathrm{LEE}}} \boldsymbol{\psi}^{\dagger} \boldsymbol{F} \boldsymbol{q} \mathrm{d} \Gamma=0,
$$

where $\boldsymbol{F}=\boldsymbol{A} n_{x}+\boldsymbol{B} n_{y}+\boldsymbol{C} n_{z}$ is the normal flux matrix, and $\boldsymbol{n}=\left(n_{x}, n_{y}, n_{z}\right)$ is the unit outward normal vector on $\mathrm{d} \Gamma$.

The boundary conditions on $\Gamma_{\mathrm{LEE}}$ are prescribed through a decomposition of the normal flux $\boldsymbol{F}$ into characteristic waves. The main idea of this flux splitting method is to separate incoming and outgoing waves [11]. The waves 
entering the LEE domain are imposed whereas the exiting waves are left free. The characteristics are defined by the eigenvalues and eigenvectors of the normal flux matrix $\boldsymbol{F}$ :

$$
\boldsymbol{F}=\boldsymbol{W} \mathbf{\Lambda} \boldsymbol{W}^{-1},
$$

with

$$
\boldsymbol{\Lambda}=\operatorname{diag}\left(\boldsymbol{u}_{0} \cdot \boldsymbol{n}, \boldsymbol{u}_{0} \cdot \boldsymbol{n}, \boldsymbol{u}_{0} \cdot \boldsymbol{n}, \boldsymbol{u}_{0} \cdot \boldsymbol{n}-c_{0}, \boldsymbol{u}_{0} \cdot \boldsymbol{n}+c_{0}\right) \quad \text { and } \boldsymbol{W}=\left[\begin{array}{ccccc}
0 & 0 & 1 & 1 & 1 \\
\boldsymbol{\tau}_{1} & \boldsymbol{\tau}_{2} & \boldsymbol{u}_{0} & \boldsymbol{u}_{0}-c_{0} \boldsymbol{n} & \boldsymbol{u}_{0}+c_{0} \boldsymbol{n} \\
0 & 0 & 0 & p_{\mathrm{c} 0} / \rho_{0} & p_{\mathrm{c} 0} / \rho_{0}
\end{array}\right] \text {, }
$$

where $p_{\mathrm{c} 0}=\left(p_{0} / p_{\infty}\right)^{1 / \gamma} . \tau_{1}$ and $\tau_{2}$ are two unit orthogonal vectors tangential to the surface $\Gamma_{\mathrm{LEE}}$. The eigenvalues in $\boldsymbol{\Lambda}$ are the phase speeds of the different characteristic waves. The flux matrix $\boldsymbol{F}$ can be decomposed into two flux matrices $\boldsymbol{F}^{+}$and $\boldsymbol{F}^{-}$containing the outgoing and incoming characteristics, respectively. It yields:

$$
\boldsymbol{F}=\boldsymbol{F}^{+}+\boldsymbol{F}^{-}=\boldsymbol{W} \boldsymbol{\Lambda}^{+} \boldsymbol{W}^{-1}+\boldsymbol{W} \boldsymbol{\Lambda}^{-} \boldsymbol{W}^{-1},
$$

where the diagonal matrices $\boldsymbol{\Lambda}^{ \pm}$contain either the positive or negative eigenvalues. The norm flux $\boldsymbol{F} \boldsymbol{q}$ can then be written as follows:

$$
\boldsymbol{F} \boldsymbol{q}=\boldsymbol{W} \boldsymbol{\Lambda}^{+} \boldsymbol{W}^{-1} \boldsymbol{q}+\boldsymbol{W} \boldsymbol{\Lambda}^{-} \boldsymbol{W}^{-1} \boldsymbol{q}=\boldsymbol{W} \boldsymbol{\Lambda}^{+} \hat{\boldsymbol{q}}+\boldsymbol{W} \boldsymbol{\Lambda}^{-} \hat{\boldsymbol{q}}
$$

where $\hat{\boldsymbol{q}}=\boldsymbol{W}^{-1} \boldsymbol{q}=\left[\hat{q}_{\mathrm{h} 1}, \hat{q}_{\mathrm{h} 2}, \hat{q}_{\mathrm{e}}, \hat{q}_{\mathrm{a}}^{-}, \hat{q}_{\mathrm{a}}^{+}\right]^{T}$ represents the amplitudes of the characteristic waves, namely the two vorticity waves $\hat{q}_{\mathrm{h} 1}$ and $\hat{q}_{\mathrm{h} 2}$, the entropy wave $\hat{q}_{\mathrm{e}}$ and the acoustic waves $\hat{q}_{\mathrm{a}}^{-}$and $\hat{q}_{\mathrm{a}}^{+}$propagating in the negative and positive directions, respectively.

The second term on the right-hand side of (8) corresponds to the characteristic waves entering the computational domain. To implement a given boundary condition, this term has to be rewritten as a function of the outgoing characteristics and any source term. For more details on this approach for the implementation of boundary conditions, the reader is referred to $[11,12]$. In Section 3 we will show how this approach is used to couple an LEE region to an LPE region.

\subsection{Linearised Potential Equation (LPE)}

For an inviscid, adiabatic, isentropic and irrotational flow, the velocity field $\boldsymbol{u}$ can be written as the gradient of a scalar potential $\phi$. The mean and fluctuating velocity vectors are thus defined as $\boldsymbol{u}_{0}=\nabla \phi_{0}$ and $\boldsymbol{u}^{\prime}=\nabla \phi^{\prime}$, respectively. The acoustic velocity potential $\phi^{\prime}$ is governed by the linearised potential equation:

$$
\rho_{0} \frac{\mathrm{D}_{0}}{\mathrm{D} t}\left(\frac{1}{c_{0}^{2}} \frac{\mathrm{D}_{0} \phi^{\prime}}{\mathrm{D} t}\right)-\nabla \cdot\left(\rho_{0} \nabla \phi^{\prime}\right)=0,
$$

where $\mathrm{D}_{0} / \mathrm{D} t=\mathrm{i} \omega+\boldsymbol{u}_{0} \cdot \nabla$ is the material derivative, $c_{0}$ is the speed of sound and $\rho_{0}$ is the mean flow density. For a computational domain $\Omega_{\mathrm{LPE}}$ with boundary $\Gamma_{\mathrm{LPE}}$, the variational formulation reads:

$$
\int_{\Omega_{\mathrm{LPE}}}\left(\frac{\rho_{0}}{c_{0}^{2}} \frac{\overline{\mathrm{D}_{0} \psi}}{\mathrm{D} t} \frac{\mathrm{D}_{0} \phi^{\prime}}{\mathrm{D} t}+\rho_{0} \nabla \bar{\psi} \cdot \nabla \phi^{\prime}\right) \mathrm{d} \Omega+\int_{\Gamma_{\mathrm{LPE}}} \frac{\rho_{0}}{c_{0}^{2}} \bar{\psi}\left(\boldsymbol{u}_{0} \cdot \boldsymbol{n}\right) \frac{\mathrm{D}_{0} \phi^{\prime}}{\mathrm{D} t}-\rho_{0} \bar{\psi} \frac{\partial \phi^{\prime}}{\partial n} \mathrm{~d} \Gamma=0,
$$

where $\psi$ is the test function, - is the complex conjugate and $\boldsymbol{n}$ is the unit outward normal vector on $\mathrm{d} \Gamma$.

To better understand how the boundary conditions can be applied it is useful to develop the boundary integral as follows:

$$
\int_{\Gamma_{\mathrm{LPE}}} \frac{\rho_{0}}{c_{0}^{2}} \bar{\psi}\left(\boldsymbol{u}_{0} \cdot \boldsymbol{n}\right) \frac{\mathrm{D}_{0} \phi^{\prime}}{\mathrm{D} t}-\rho_{0} \bar{\psi} \frac{\partial \phi^{\prime}}{\partial n} \mathrm{~d} \Gamma=\int_{\Gamma_{\mathrm{LPE}}} \frac{\rho_{0}}{c_{0}^{2}} \bar{\psi}\left(\mathrm{i} \omega\left(\boldsymbol{u}_{0} \cdot \boldsymbol{n}\right) \phi^{\prime}+\left(\left(\boldsymbol{u}_{0} \cdot \boldsymbol{n}\right)^{2}-c_{0}^{2}\right) \frac{\partial \phi^{\prime}}{\partial n}+\left(\boldsymbol{u}_{0} \cdot \boldsymbol{n}\right) \boldsymbol{u}_{0 \perp} \cdot \nabla_{\perp} \phi^{\prime}\right) \mathrm{d} \Gamma,
$$

where $\boldsymbol{u}_{0 \perp}=\boldsymbol{u}_{0}-\left(\boldsymbol{u}_{0} \cdot \boldsymbol{n}\right) \boldsymbol{n}$ is the part of $\boldsymbol{u}_{0}$ that is tangential to the surface $\Gamma_{\mathrm{LPE}}$. The gradient $\nabla_{\perp}$ operates only in the plane tangential to the surface (i.e. along the directions $\tau_{1}$ and $\tau_{2}$ ).

In order to impose a boundary condition for the LPE, it is necessary to replace the normal derivative of $\phi^{\prime}$ by the appropriate terms involving $\phi^{\prime}, \nabla_{\perp} \phi^{\prime}$ or known quantities. For instance, for a vibrating surface, one would write 
$\boldsymbol{u}_{0} \cdot \boldsymbol{n}=0$ and $\partial \phi^{\prime} / \partial n=V$ where $V$ is the imposed normal velocity. In the next section, we will see how to calculate $\partial \phi^{\prime} / \partial n$ to couple an LPE region to an LEE region.

Finally, Perfectly Matched Layers (PML) are used around the boundary of the computational domain to absorb any outgoing waves and to represent sound radiation to the far field. The PML can also be used to generate a sound field entering the computational domain, such as duct modes. The reader is referred to references [13, 14] for further details.

\section{Coupling of LEE and LPE propagation models}

The coupling between the LEE and LPE involves finding well-posed boundary conditions at the interface between the LEE and the LPE regions. For this purpose, we follow an approach similar to that proposed in [15]. The coupling method proposed in this paper is explained for the configuration shown in Figure 1. The domain $\Omega$ is composed of two non-overlapping subdomains, namely the LEE subdomain $\Omega_{\mathrm{LEE}}$ and the LPE subdomain $\Omega_{\mathrm{LPE}}$. The subdomain boundaries are denoted $\Gamma_{\mathrm{LEE}}$ and $\Gamma_{\mathrm{LPE}}$, with the unit outward normal vectors $\boldsymbol{n}_{\mathrm{LEE}}$ and $\boldsymbol{n}_{\mathrm{LPE}}$.

As explained above, for the LEE we need to specify the characteristic waves propagating into the domain $\Omega_{\mathrm{LEE}}$. 65 The number of incoming characteristics depends on $\boldsymbol{u}_{0} \cdot \boldsymbol{n}_{\mathrm{LEE}}$ and we have to consider two distinct cases. Note that in the following we assume a subsonic base flow $\left(\left|\boldsymbol{u}_{0}\right|<c_{0}\right)$.

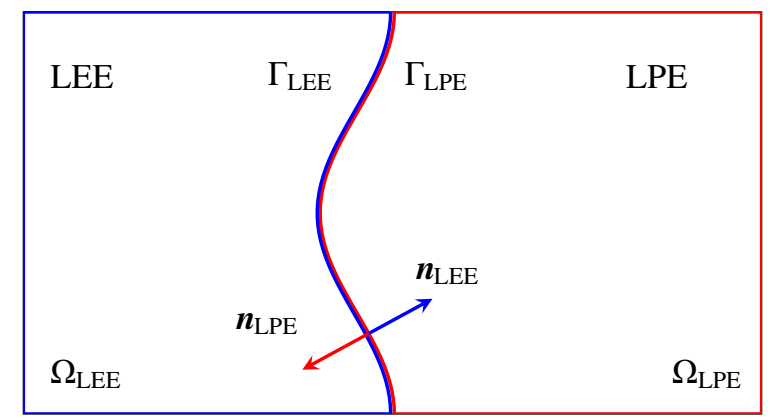

Figure 1: Rectangular domain $\Omega$ composed of two subdomains $\Omega_{\mathrm{LEE}}$ and $\Omega_{\mathrm{LPE}}$.

\subsection{Outflow $\left(\boldsymbol{u}_{0} \cdot \boldsymbol{n}_{\mathrm{LEE}}>0\right)$}

If at a point on the boundary $\Gamma_{\mathrm{LEE}}$ the flow is going from the LEE region into the LPE region, i.e. $\boldsymbol{u}_{0} \cdot \boldsymbol{n}_{\mathrm{LEE}}>0$, there is only one characteristic wave propagating into the LEE domain. Namely, only the acoustic characteristic $\hat{q}_{\mathrm{a}}^{-}$, which has a negative phase speed $\boldsymbol{u}_{0} \cdot \boldsymbol{n}_{\mathrm{LEE}}-c_{0}$, needs to be specified. In addition, for the LPE region, we need to rewrite the normal derivative of $\phi^{\prime}$. We therefore need two coupling conditions at the interface $\Gamma_{\mathrm{LEE}}$. The continuity of pressure and normal velocity are imposed for this purpose:

$$
p_{\mathrm{LEE}}^{\prime}=p_{\mathrm{LPE}}^{\prime}, \quad \boldsymbol{u}_{\mathrm{LEE}}^{\prime} \cdot \boldsymbol{n}_{\mathrm{LPE}}=\boldsymbol{u}_{\mathrm{LPE}}^{\prime} \cdot \boldsymbol{n}_{\mathrm{LPE}} .
$$

The rationale for choosing these conditions is that they involve the quantities (pressure and normal velocity) that are needed on a surface to fully specify the acoustic field using Kirchhoff's integral theorem.

We have to rewrite the LEE quantities in terms of the characteristic wave amplitudes $\hat{\boldsymbol{q}}$. For this purpose, we use $\boldsymbol{q}=\boldsymbol{W} \hat{\boldsymbol{q}}$ together with equation (6) to write:

$$
\begin{aligned}
\rho_{\mathrm{LEE}}^{\prime} & =\hat{q}_{\mathrm{e}}+\hat{q}_{\mathrm{a}}^{-}+\hat{q}_{\mathrm{a}}^{+}, \\
(\rho \boldsymbol{u})_{\mathrm{LEE}}^{\prime} & =\boldsymbol{\tau}_{1} \hat{q}_{\mathrm{h} 1}+\boldsymbol{\tau}_{2} \hat{q}_{\mathrm{h} 2}+\boldsymbol{u}_{0} \hat{q}_{\mathrm{e}}+\left(\boldsymbol{u}_{0}-c_{0} \boldsymbol{n}\right) \hat{q}_{\mathrm{a}}^{-}+\left(\boldsymbol{u}_{0}+c_{0} \boldsymbol{n}\right) \hat{q}_{\mathrm{a}}^{+}, \\
p_{\mathrm{c}, \mathrm{LEE}}^{\prime} & =\frac{p_{\mathrm{c} 0}}{\rho_{0}}\left(\hat{q}_{\mathrm{a}}^{+}+\hat{q}_{\mathrm{a}}^{-}\right) .
\end{aligned}
$$


Conversely, the LPE quantities should be rewritten in terms of the velocity potential $\phi^{\prime}$ as:

$$
p_{\mathrm{LPE}}^{\prime}=-\rho_{0} \frac{\mathrm{D}_{0} \phi^{\prime}}{\mathrm{D} t}, \quad \boldsymbol{u}_{\mathrm{LPE}}^{\prime} \cdot \boldsymbol{n}_{\mathrm{LPE}}=\frac{\partial \phi^{\prime}}{\partial n_{\mathrm{LPE}}} .
$$

We now substitute these expressions in (12) to get:

$$
\begin{gathered}
c_{0}^{2}\left(\hat{q}_{\mathrm{a}}^{+}+\hat{q}_{\mathrm{a}}^{-}\right)=-\rho_{0}\left[\mathrm{i} \omega \phi^{\prime}+\left(\boldsymbol{u}_{0} \cdot \boldsymbol{n}_{\mathrm{LPE}}\right) \frac{\partial \phi^{\prime}}{\partial n_{\mathrm{LPE}}}+\boldsymbol{u}_{0 \perp} \cdot \nabla_{\perp} \phi^{\prime}\right], \\
\frac{c_{0}}{\rho_{0}}\left(\hat{q}_{\mathrm{a}}^{+}-\hat{q}_{\mathrm{a}}^{-}\right)=-\frac{\partial \phi^{\prime}}{\partial n_{\mathrm{LPE}}} .
\end{gathered}
$$

These two equations can then be rearranged to obtain the required quantities:

$$
\begin{aligned}
\hat{q}_{\mathrm{a}}^{-} & =\frac{\boldsymbol{u}_{0} \cdot \boldsymbol{n}_{\mathrm{LEE}}+c_{0}}{\boldsymbol{u}_{0} \cdot \boldsymbol{n}_{\mathrm{LEE}}-c_{0}} \hat{q}_{\mathrm{a}}^{+}+\frac{\rho_{0} / c_{0}}{\boldsymbol{u}_{0} \cdot \boldsymbol{n}_{\mathrm{LEE}}-c_{0}}\left(\mathrm{i} \omega \phi^{\prime}+\boldsymbol{u}_{0 \perp} \cdot \nabla_{\perp} \phi^{\prime}\right), \\
\frac{\partial \phi^{\prime}}{\partial n_{\mathrm{LPE}}} & =\frac{-2 c_{0}^{2} / \rho_{0}}{\boldsymbol{u}_{0} \cdot \boldsymbol{n}_{\mathrm{LPE}}+c_{0}} \hat{q}_{\mathrm{a}}^{+}-\frac{1}{\boldsymbol{u}_{0} \cdot \boldsymbol{n}_{\mathrm{LPE}}+c_{0}}\left(\mathrm{i} \omega \phi^{\prime}+\boldsymbol{u}_{0 \perp} \cdot \nabla_{\perp} \phi^{\prime}\right) .
\end{aligned}
$$

We can use the above expression for $\hat{q}_{\mathrm{a}}^{-}$to specify the second term on the right-hand side of equation (8). We can also rewrite the boundary integral for the LPE region on the boundary with the LEE region:

$$
\int_{\Gamma_{\mathrm{LPE}}} \frac{\rho_{0}}{c_{0}^{2}} \bar{\psi}\left(\boldsymbol{u}_{0} \cdot \boldsymbol{n}_{\mathrm{LPE}}\right) \frac{\mathrm{D}_{0} \phi^{\prime}}{\mathrm{D} t}-\rho_{0} \bar{\psi} \frac{\partial \phi^{\prime}}{\partial n} \mathrm{~d} \Gamma=\int_{\Gamma_{\mathrm{LPE}}}-2\left(\boldsymbol{u}_{0} \cdot \boldsymbol{n}_{\mathrm{LPE}}-c_{0}\right) \bar{\psi} \hat{q}_{\mathrm{a}}^{+}+\frac{\rho_{0}}{c_{0}} \bar{\psi}\left(\mathrm{i} \omega \phi^{\prime}+\boldsymbol{u}_{0 \perp} \cdot \nabla_{\perp} \phi^{\prime}\right) \mathrm{d} \Gamma,
$$

70 3.2. Inflow $\left(\boldsymbol{u}_{0} \cdot \boldsymbol{n}_{\mathrm{LEE}}<0\right)$

When the mean flow is going from the LPE region into the LEE region, there are four characteristics waves that enter the LEE region, and that need to be specified, namely $\hat{q}_{\mathrm{h} 1}, \hat{q}_{\mathrm{h} 2}, \hat{q}_{\mathrm{e}}$ and $\hat{q}_{\mathrm{a}}^{-}$. In addition, we have to specify $\partial \phi^{\prime} / \partial n$, so we have to prescribe five conditions on the interface between the two regions. We impose the continuity of all the field variables on $\Gamma_{\mathrm{LPE}}$, namely: density, velocity and pressure:

$$
\rho_{\mathrm{LEE}}^{\prime}=\rho_{\mathrm{LPE}}^{\prime}, \quad \boldsymbol{u}_{\mathrm{LEE}}^{\prime}=\boldsymbol{u}_{\mathrm{LPE}}^{\prime}, \quad p_{\mathrm{LEE}}^{\prime}=p_{\mathrm{LPE}}^{\prime}
$$

which provides exactly the right number of conditions on the interface.

Firstly, we can note that, using the continuity of pressure and normal velocity, we can follow the same derivation as shown above to obtain the conditions (17) and (18). Therefore, these two conditions apply irrespective of the direction of the mean flow.

Secondly, to specify the amplitude of the entropy characteristic wave, we note that in the LPE region $p_{\mathrm{LPE}}^{\prime}=c_{0}^{2} \rho_{\mathrm{LPE}}^{\prime}$. It then follows from equation (20) that $p_{\mathrm{LEE}}^{\prime}=c_{0}^{2} \rho_{\mathrm{LEE}}^{\prime}$. Using equations (13a) and (13c) it is straightforward to show that $\hat{q}_{\mathrm{e}}=0$. This is expected since there is no entropy wave in the LPE region.

Finally, to specify the amplitudes of the two vorticity waves we use the remaining two conditions from (20) concerning the continuity of the tangential components of velocity:

$$
u_{\mathrm{LEE}}^{\prime} \cdot \tau_{1}=u_{\mathrm{LPE}}^{\prime} \cdot \tau_{1}, \quad u_{\mathrm{LEE}}^{\prime} \cdot \tau_{2}=u_{\mathrm{LPE}}^{\prime} \cdot \tau_{2}
$$

Projecting (13b) on the vectors $\tau_{1}$ and $\tau_{2}$ we get:

$$
(\rho \boldsymbol{u})_{\mathrm{LEE}}^{\prime} \cdot \boldsymbol{\tau}_{1}=\hat{q}_{\mathrm{h} 1}+\left(\boldsymbol{u}_{0} \cdot \boldsymbol{\tau}_{1}\right)\left(\hat{q}_{\mathrm{a}}^{+}+\hat{q}_{\mathrm{a}}^{-}\right), \quad(\rho \boldsymbol{u})_{\mathrm{LEE}}^{\prime} \cdot \boldsymbol{\tau}_{2}=\hat{q}_{\mathrm{h} 2}+\left(\boldsymbol{u}_{0} \cdot \boldsymbol{\tau}_{2}\right)\left(\hat{q}_{\mathrm{a}}^{+}+\hat{q}_{\mathrm{a}}^{-}\right) .
$$

Subtracting $\rho_{\mathrm{LEE}}^{\prime} \boldsymbol{u}_{0} \cdot \boldsymbol{\tau}_{1}$ and $\rho_{\mathrm{LEE}}^{\prime} \boldsymbol{u}_{0} \cdot \tau_{2}$ to either of these equations, using (13a) and recalling that we found $\hat{q}_{\mathrm{e}}=0$ lead to:

$$
\begin{aligned}
& \hat{q}_{\mathrm{h} 1}=\rho_{0} \boldsymbol{u}_{\mathrm{LEE}}^{\prime} \cdot \boldsymbol{\tau}_{1}=\rho_{0} \boldsymbol{u}_{\mathrm{LPE}}^{\prime} \cdot \tau_{1}=\rho_{0} \frac{\partial \phi^{\prime}}{\partial \tau_{1}}, \\
& \hat{q}_{\mathrm{h} 2}=\rho_{0} \boldsymbol{u}_{\mathrm{LEE}}^{\prime} \cdot \boldsymbol{\tau}_{2}=\rho_{0} \boldsymbol{u}_{\mathrm{LPE}}^{\prime} \cdot \tau_{2}=\rho_{0} \frac{\partial \phi^{\prime}}{\partial \tau_{2}} .
\end{aligned}
$$


In practice, the vectors $\tau_{1}$ and $\tau_{2}$ are taken in the LPE domain in order to easily compute the derivatives of $\phi^{\prime}$ in equations (22):

$$
\tau_{1}=\tau_{1 \mathrm{LPE}}, \quad \tau_{2}=\tau_{2 \mathrm{LPE}}
$$

The incoming vorticity waves into the LEE region are therefore defined in terms of the tangential derivatives of the velocity potential in the LPE region. It could seem counterintuitive to induce vorticity waves in the LEE region from an LPE region where there is no vorticity. But attempting to use $\hat{q}_{\mathrm{h} 1}=\hat{q}_{\mathrm{h} 2}=0$ in the test case shown in Section 5.1 resulted in less accurate results than the conditions derived above.

\section{High-Order Finite Element Model}

\subsection{Presentation of $p-F E M$}

The coupled variational formulations (4) and (10) are discretized using a hierarchical $H^{1}$-conforming high-order finite element method based on Lobatto shape functions. The details of this finite element method (FEM) are not presented here and only its key features are recalled. For an exhaustive description of the numerical scheme, the reader is referred to the references $[8,9]$ as well as the textbooks $[16,17]$. This high-order FEM provides significant reductions in memory and CPU time when compared to conventional finite element models [18, 8] and has been shown

90 to deliver comparable, and in some cases even superior, performance compared to high-order Trefftz methods on a variety of Helmholtz problems $[19,20]$. The benefits of $p$-FEM (e.g. low dispersion error, exponential $p$-convergence) are also retained in the presence of background mean flows [21].

A key property of the high-order FEM is that it is possible to eliminate the degrees of freedom associated with the bubble shape functions since these degrees of freedom do not interact with neighboring elements [17]. This technique, called condensation, allows to reduce the size of the global linear system before it is assembled and solved, hence reducing the memory requirements. The $p$-FEM shape functions provide an additional benefit. Their 'hierarchic'

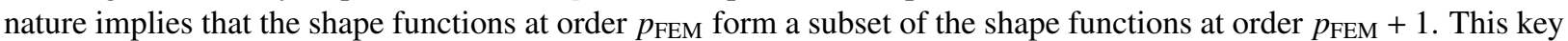
property implies that $p$-FEM can easily handle local order variations: two adjacent elements can coexist with different polynomial orders. This is in contrast with high-order Lagrange interpolation functions which cannot handle easily different orders for two contiguous elements. To ensure the continuity of the numerical solution, the order attributed to a given edge or face shared by several elements is chosen based on a so-called conformity rule [16].

\subsection{A priori error indicator}

For an improved efficiency, $p$-adaptive solutions are considered in this work. The element order distribution is automatically assigned at each frequency, prior to the calculation, based on the flow properties and the element size. Bériot et al. introduced an efficient a-priori error indicator for Helmholtz problem solved with $p$-FEM [8]. With this approach, the numerical error incurred on a given element is estimated based on the numerical error measured on a single, one-dimensional element with representative wavenumber $k$ and length $h$. Despite its apparent simplicity, this indicator was shown to efficiently control the global numerical error in realistic two-dimensional and three-dimensional problems with strong mesh non-uniformities. The adaptive approach consists in selecting the interpolation order $p_{\text {FEM }}$ in each element so that the $L^{2}$ interpolation error remains below a user-defined target error $E_{T}$ in the 1D element. In practice, one does not need to solve the 1D model for every element of the mesh, since the order $p\left(k h, E_{T}\right)$ required to have an actual error below the target $E_{T}$ can be tabulated in terms of the mesh resolution $k h$ and the error level $E_{T}$ (see [8] for more details). This method was later extended to the linearised potential equation model in [9] and to the linearised Euler equations in [6]. Recently, a novel version of this error indicator was introduced, which enables anisotropic $p$-refinement [22]. This strategy allows to control the error on elements with strong distortion and, in turn, makes the simulation largely independent of badly shaped elements in the mesh. In addition, anisotropic $p$-refinement also provides significant cost reductions for flow acoustics applications, where the wave properties are strongly direction dependent.

We discuss here the application of the error indicator to coupled LEE- LPE problems. This is a non-trivial issue, because the time harmonic perturbations described in the LEE do not only contain acoustic waves but also include vorticity and entropy waves. 
In two dimensions, the dispersion relations for the acoustic, hydrodynamic and entropy waves read [23]:

$$
\mathbf{k}_{\mathrm{a}, \mathrm{h}, \mathrm{e}}=k_{\mathrm{a}, \mathrm{h}, \mathrm{e}}\left(\begin{array}{c}
\cos \theta_{\mathrm{w}} \\
\sin \theta_{\mathrm{w}}
\end{array}\right), \quad \text { with } k_{\mathrm{a}}=\frac{k_{0}}{1+M_{0} \cos \left(\theta_{\mathrm{w}}-\theta_{0}\right)}, \quad k_{\mathrm{h}}=k_{\mathrm{e}}=\frac{k_{0}}{M_{0} \cos \left(\theta_{\mathrm{w}}-\theta_{0}\right)},
$$

where $\mathbf{k}$ is the wavenumber vector, $\theta_{\mathrm{w}}$ designates the plane wave orientation and $k_{0}=\omega / c_{0}$. The direction and velocity of the mean flow are given by the angle $\theta_{0}$ and the Mach number $M_{0}=\left|\boldsymbol{u}_{0}\right| / c_{0}$. Note that the entropy and hydrodynamic waves, which are purely convected, are ruled by the same dispersion relation.

The different wavenumber vectors obtained for a Mach number $M_{0}=0.5$ and a mean flow orientation $\theta_{0}=\pi / 4$ are illustrated in Figure 2(a). For acoustic waves $\mathbf{k}_{\mathrm{a}}$, represented by gray arrows, the Doppler effect induced by the presence of the mean flow is clearly visible: the wavenumber decreases for downstream propagation (when $\cos \left(\theta_{\mathrm{w}}-\theta_{0}\right)>0$ ) and increases for upstream propagation (when $\cos \left(\theta_{\mathrm{w}}-\theta_{0}\right)<0$ ). This corresponds to longer and shorter wavelengths, respectively. Hydrodynamic and entropy waves, on the other hand, indicated by turquoise arrows, are propagating in the direction of the flow. Their accurate discretization poses severe difficulties. In particular, in the limit case where they propagate almost perpendicularly to the mean flow, i.e. for small values of $\left|\theta_{\mathrm{w}}-\theta_{0}\right|$, their wavelength tends to zero.

(a) Wave vectors at $M_{0}=0.5, \theta_{0}=\pi / 4$

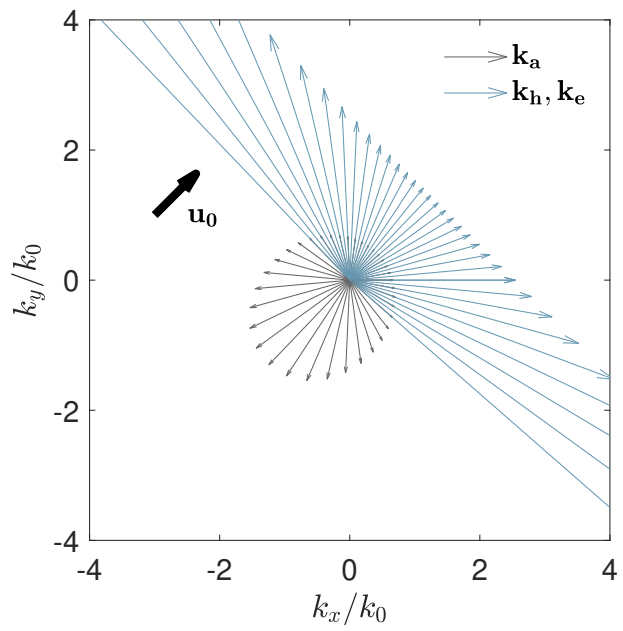

(b) LEE normalized length scales

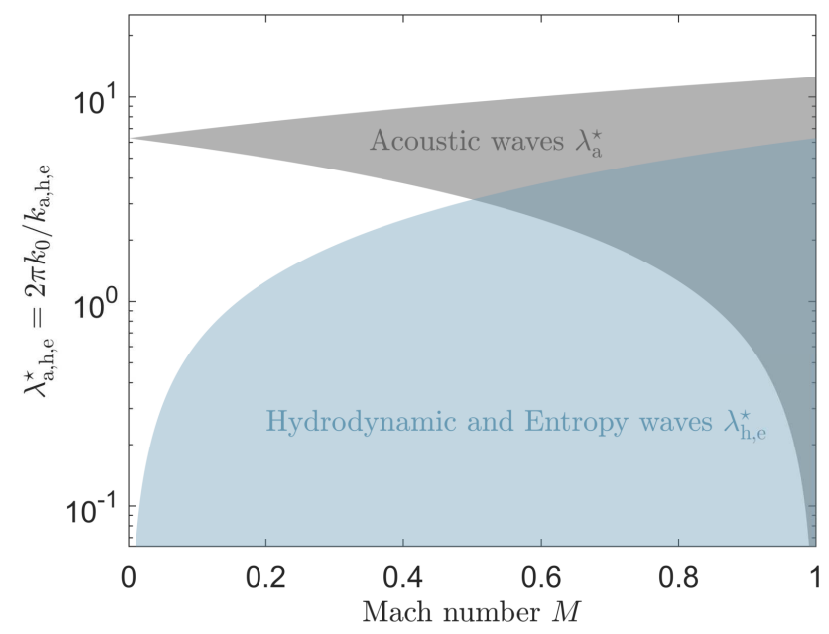

Figure 2: (a) Acoustic (gray) and hydrodynamic/entropy (turquoise) wave vectors normalized by $k_{0}=\omega / c_{0}$ for a given mean flow magnitude and orientation (symbolized by the black arrow). (b) Range of normalized length scales $\lambda_{\mathrm{a}, \mathrm{h}, \mathrm{e}}^{\star}=2 \pi k_{0} / k_{\mathrm{a}, \mathrm{h}, \mathrm{e}}$ obtained from the dispersion relations (24), as a function of the Mach number.

Figure 2(b) presents the normalized admissible wavelengths (defined as $\lambda_{\mathrm{a}, \mathrm{h}, \mathrm{e}}^{\star}=\lambda_{\mathrm{a}, \mathrm{h}, \mathrm{e}} k_{0}=2 \pi k_{0} / k$ ) obtained from the dispersion relations (24), in logarithmic scale, as a function of the Mach number. From this representation, the LEE may in fact be seen as a multi-scale operator, supporting multiple physical features co-existing at different length scales. As is known, in the presence of sheared flows, the acoustic disturbances are not irrotational anymore and become coupled with the hydrodynamic disturbances due to entropy and vorticity waves [24]. However, these vortical solutions present a wide range of length scales $\left.\left.\lambda_{\mathrm{h}, \mathrm{e}}^{\star}=\right] 0,2 \pi M_{0}\right]$. The resolution of the smallest structures (corresponding to close-to perpendicular propagation and/or to regions approaching no-flow conditions) would rapidly lead to computationally intractable solutions.

Beyond these practical cost considerations, a more fundamental issue is that hydrodynamic instabilities, of KelvinHelmholtz (K-H) type, may be present [25]. With linear models like the LEE, the K-H instabilities exhibit an exponential growth which may, in some occasions, completely obscure the acoustic solution downstream of the source. This has led to the development of dedicated mitigation strategies, such as the gradient term suppression (GTS) method [26], to avoid the development of vorticity shedding at the trailing edge and suppress the hydrodynamic instabilities from the computed solution. However, this type of methods modify the original LEE formulation, which may change the physics of the sound interaction with the vorticity field. Computing the solution in the frequency 
domain allows the suppression of the temporal growth of the instability waves [27], but a spatially growing vortical wave is generally still observed, and it is difficult to infer to which extent it is physical or not. Previous numerical works have investigated the behaviour of the LEE solutions formulated in the frequency domain. In [5], Özyörük and Tester examined the sound scattering through a realistic jet flow, by performing successive $h$-refinements of the grid. They concluded that the sound radiation pattern is not altered noticeably by the development of the hydrodynamic instability. However, they also argue that a 2-3 dB amplification effect should be expected on the radiated peak pressure levels, when allowing the development of the instability waves in the shear layer. In Iob et al. [28], the grid size for the time-harmonic LEE is chosen based solely on the acoustic dispersion properties. Although no instability waves are observed in the jet mixing region (as they fall beneath the grid resolution limits) satisfactory results were obtained compared to a semi-analytical reference model. Finally, in [6], the radiated far-field acoustic pressure is shown to be almost invariant to successive $p$-refinements applied in the shear-layer region.

What these previous studies suggest is that resolving the full range of acoustic admissible wavelengths (corresponding to the gray region in Figure 2(b), namely $\lambda_{\mathrm{a}}^{\star}=2 \pi\left[1-M_{0}, 1+M_{0}\right]$ ) is sufficient to capture the main characteristics of the sound radiation through a sheared flow. In this work, we therefore apply the same approach. The element order is determined solely based on the dispersion properties of the acoustic waves, invariably in the LEE and LPE domains, using the a priori error indicator from reference [22]. Unless a strong grid refinement is applied in the shear layer region, this strategy amounts to neglecting the influence of sub-acoustic wavelength structures on the sound propagation.

\section{Numerical verification}

In order to verify the coupling formulation presented in Section 3 and to evaluate the performance of the proposed hybrid LEE-LPE numerical model, two three-dimensional test cases are examined. The first one considers the propagation of a set of acoustic plane waves in a uniform mean flow while the second one focuses on the sound scattering through a strongly sheared non-isothermal flow.

All the computations reported hereafter were performed on a Dell Poweredge R730XD equipped with two Intel Xeon E5-2667 CPUs with 16 cores running at $3.20 \mathrm{GHz}$ and with $384 \mathrm{~GB}$ of memory. The unstructured meshes are prepared using the open source mesh generator Gmsh [29]. The matrices are assembled using an in-house adaptive $p$-FEM research code developed in Matlab (the assembly timings are therefore not reported). The resulting sparse linear system of equations is solved sequentially using the fast, multi-frontal solver MUMPS v5.0.1 [30].

\subsection{Propagation in uniform flow}

The first test case consists of a cube where time-harmonic plane waves propagate in the presence of a uniform mean flow defined by a Mach number $M_{0}=u_{0} / c_{0}=0.9$, a density $\rho_{0}=1.225 \mathrm{~kg} / \mathrm{m}^{3}$ and a speed of sound $c_{0}=340 \mathrm{~m} / \mathrm{s}$. The computational domain is displayed in Figure 3(a). The cube has a side $l_{\mathrm{c}}=2 \mathrm{~m}$ and is centered on $\boldsymbol{x}_{0}=(0,0,0)^{T}$. It is decomposed into an LEE region and an LPE region that do not overlap. The LEE region is the sphere of center $\boldsymbol{x}_{0}$ and radius $R=0.25 l_{\mathrm{c}}$. The LPE region is the volume outside the sphere. In this study, the mean flow is oriented in the $x$ direction, i.e. $\boldsymbol{u}_{0}=\left(u_{0}, 0,0\right)^{T}$. A total of 36 plane waves are simulated separately in these calculations. Each plane wave is defined by the scalar potential:

$$
\phi_{\text {analytic }}^{\prime}=A \exp (-\mathrm{i} \boldsymbol{k} \cdot \boldsymbol{x}) \quad \text { with } \quad \boldsymbol{k}=k(\cos \theta \cos \alpha, \sin \theta \cos \alpha, \sin \alpha) \quad \text { and } \quad k=\frac{k_{0}}{1+M_{0} \cos \alpha \cos \theta},
$$

where $A$ is the wave amplitude, and $\theta$ and $\alpha$ are the wave directions according to the reference frame of Figure 3(b). The angles $\theta$ and $\alpha$ are uniformly distributed over the ranges $\theta \in[0,2 \pi]$ and $\alpha \in[0, \pi]$, with $\Delta \theta=2 \pi / 5$ and $\Delta \alpha=\pi / 5$. The value of $A$ is arbitrarily set to $A=100 \mathrm{~m}^{2} / \mathrm{s}$. The FEM simulations are carried out with an unstructured mesh composed of tetrahedral linear elements of size $h=l_{\mathrm{c}} / 8$. The mesh contains 4224 elements in the LPE region and 453 elements in the LEE region. The frequency range for the simulations is chosen so that the normalized wavenumber $k_{0} h$ varies over the range $[0.01,10]$. For each simulation, the polynomial order $p_{\text {FEM }}$ is constant in the computational domain. Simulations are carried out with polynomial orders $p_{\text {FEM }}$ varying from 1 to 6. 
(a)

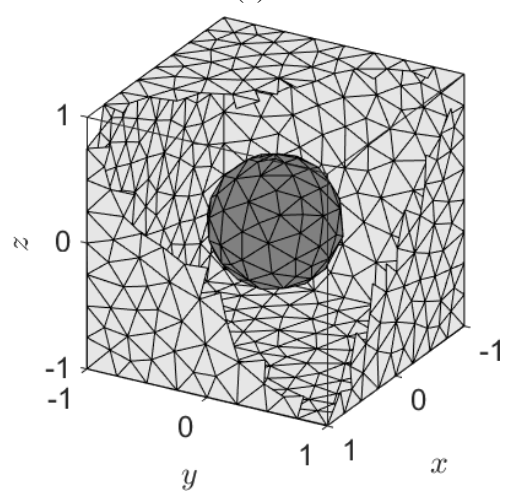

(b)

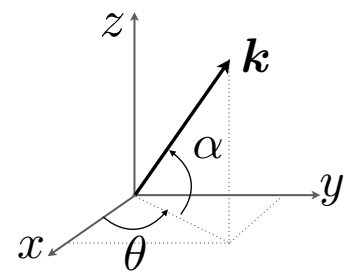

Figure 3: Representation of (a) the computational domain: LEE region inside the sphere and LPE region outside, and (b) the coordinate system used for the definition of the plane waves.

The objective is to evaluate the influence of the LPE-LEE coupling on the pressure computed inside the computational domain. For this purpose, the pressure $p^{\prime}$ obtained numerically is compared to the analytical solution $p_{\text {analytic }}$ through the $L_{2}$ relative error:

$$
\epsilon_{p}=\left(\frac{\int_{\Omega}\left|p^{\prime}-p_{\text {analytic }}^{\prime}\right|^{2} \mathrm{~d} \Omega}{\int_{\Omega}\left|p_{\text {analytic }}^{\prime}\right|^{2} \mathrm{~d} \Omega}\right)^{1 / 2} \quad \text { with } \quad p_{\text {analytic }}^{\prime}=-\rho_{0} \frac{\mathrm{D}_{0} \phi_{\text {analytic }}^{\prime}}{\mathrm{D} t} .
$$

In the LPE domain, the relative error is also computed for the velocity potential $\phi^{\prime}$ as:

$$
\epsilon_{\phi}=\left(\frac{\int_{\Omega}\left|\phi^{\prime}-\phi_{\text {analytic }}^{\prime}\right|^{2} \mathrm{~d} \Omega}{\int_{\Omega}\left|\phi_{\text {analytic }}^{\prime}\right|^{2} \mathrm{~d} \Omega}\right)^{1 / 2} .
$$

In practice, the relative error $\epsilon$ is evaluated in the LPE and the LEE domains for each of the 36 plane waves. In

\subsection{Propagation in a sheared non-isothermal flow}

The performance of the proposed hybrid propagation model is now verified on a problem involving a strongly sheared non-isothermal flow. The acoustic radiation from a straight circular semi-infinite duct, under hot-jet exhaust flow conditions is considered. The duct radius is $r_{\mathrm{d}}=1 \mathrm{~m}$ and the duct length is $l_{\mathrm{d}}=2.5 \mathrm{~m}$. The duct wall is 
(a)

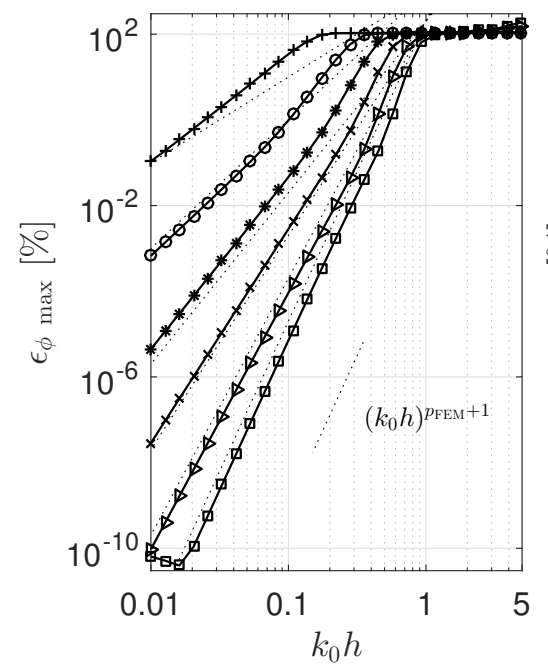

(b)

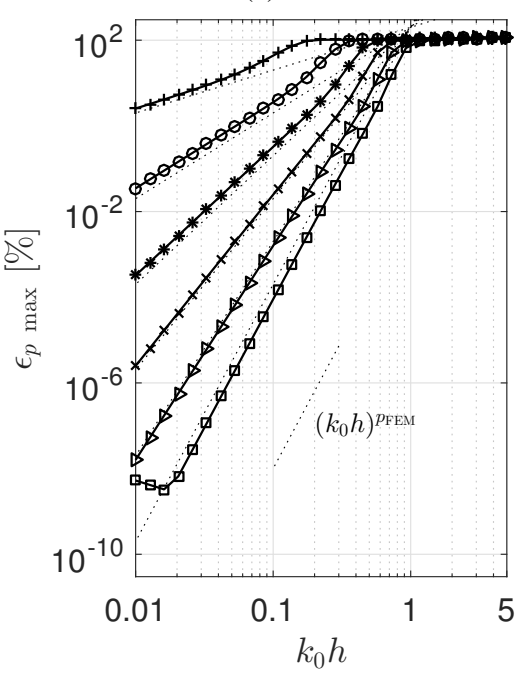

(c)

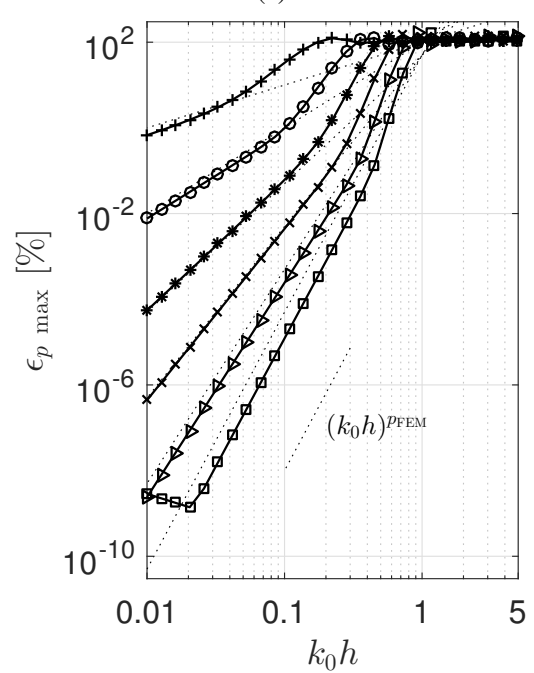

Figure 4: Maximum relative error $\epsilon_{\max }$ in the LPE domain for (a) the velocity potential $\phi^{\prime}$ and (b) the pressure $p^{\prime}$ and (c) in the LEE domain for the pressure $p^{\prime}$, as a function of the Helmholtz number $k_{0} h$, for polynomial orders $+p_{\mathrm{FEM}}=1, \circ p_{\mathrm{FEM}}=2, * p_{\mathrm{FEM}}=3, \times p_{\mathrm{FEM}}=4, \triangleright p_{\mathrm{FEM}}=5$ and $\square p_{\text {FEM }}=6$. Dotted lines: asymptotic convergence slopes.

infinitely thin and hard-wall boundary conditions are applied on both sides. Inside the duct, a uniform hot-jet flow is prescribed with $\rho_{0_{\mathrm{d}}}=0.5245 \mathrm{~kg} / \mathrm{m}^{3}, c_{0_{\mathrm{d}}}=520 \mathrm{~m} / \mathrm{s}$ and $M_{0_{\mathrm{d}}}=u_{0_{\mathrm{d}}} / c_{0_{\mathrm{d}}}=0.5$. The duct exits into a quiescent medium characterized by a density $\rho_{0_{\infty}}=1.225 \mathrm{~kg} / \mathrm{m}^{3}$, a speed of sound $c_{0_{\infty}}=340.27 \mathrm{~m} / \mathrm{s}$ and a Mach number $M_{0_{\infty}}=u_{0_{\infty}} / c_{0_{\infty}}=0$. The heat specific ratio is $\gamma=1.4$. The Mach number flow profile outside the duct is given in cylindrical coordinates by:

$$
M_{0}(r, z)=\overline{M_{0}}\left[1-\left(\frac{M_{0_{\infty}}-M_{0_{\mathrm{d}}}}{M_{0_{\infty}}+M_{0_{\mathrm{d}}}}\right) \tanh \left(\frac{r_{\mathrm{d}}-r}{\zeta \delta(z)}\right)\right],
$$

where $\overline{M_{0}}=\left(M_{0_{\mathrm{d}}}+M_{0_{\infty}}\right) / 2, \zeta=(2 / 5) \cos ^{2}(\beta / 2)$ is a parameter to control the shear layer profile, $\beta=\pi / 9$ is the spreading angle and $\delta$ is the shear layer thickness. The latter reads:

$$
\delta(z)=2\left(z-z_{\mathrm{d}}\right) \tan (\beta / 2)
$$

where $z_{\mathrm{d}}$ is the duct tip position in $z$ direction. The resulting Mach number is shown in Figure 5(a). The mean flow density is continuously varied using the same hyperbolic tangent profile. Finally, the speed of sound distribution is determined so as to maintain a constant hydrodynamic pressure $p_{0}=\rho_{0} c_{0}^{2} / \gamma=1.4183 \times 10^{5} \mathrm{~Pa}$ across the jet mixing region.

In this study, a three-dimensional computational domain $\Omega$ extending from $z=0 \mathrm{~m}$ to $z=5 \mathrm{~m}$ in the axial direction and from and $r=0$ to $r=3 \mathrm{~m}$ in the radial direction is considered. It is shown in Figure 5(a). The domain $\Omega$ is split into two non-overlapping regions: an inner subdomain $\Omega_{\mathrm{LEE}}$ enclosing the shear layer where the LEE are used, and an outer subdomain $\Omega_{\text {LPE }}$ where the LPE applies. There are a number of aspects to consider when deciding where to define the interface between these two regions:

- The LEE subdomain should be as small as possible to minimize the computational cost.

- The LEE subdomain should be sufficiently large to encompass the region where the mean flow vorticity is expected to have a significant influence on sound refraction, or where the presence of vorticity waves and entropy waves is expected.

- In this study the LEE region is not extended inside the PML and is terminated slightly before the outer boundary. This allows us to avoid reformulating the coupling conditions inside the PML, where the handling of hydrodynamic and entropy waves may pose additional difficulties. 
(a)

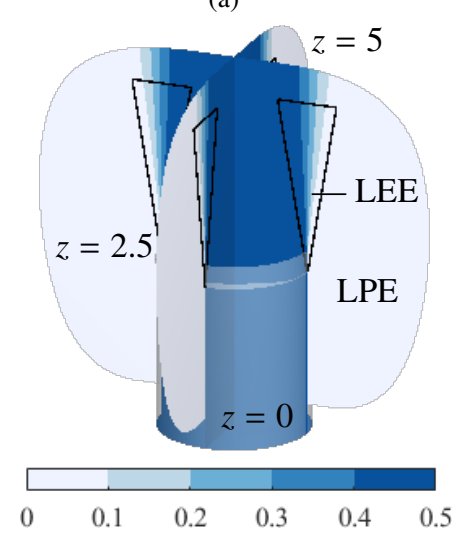

(b)

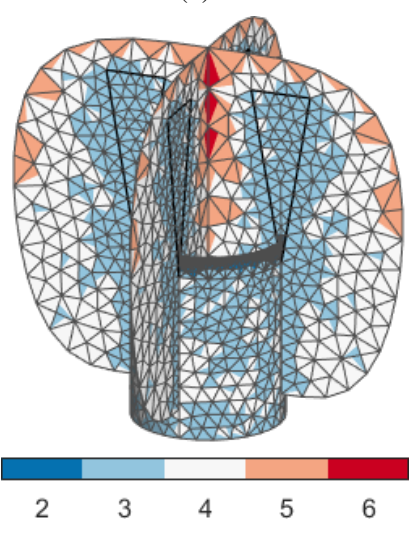

(c)

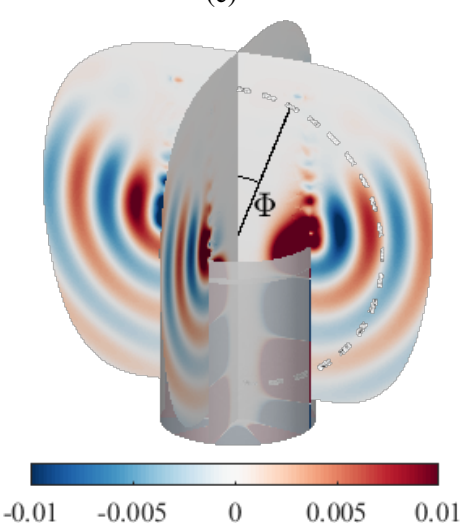

(d)

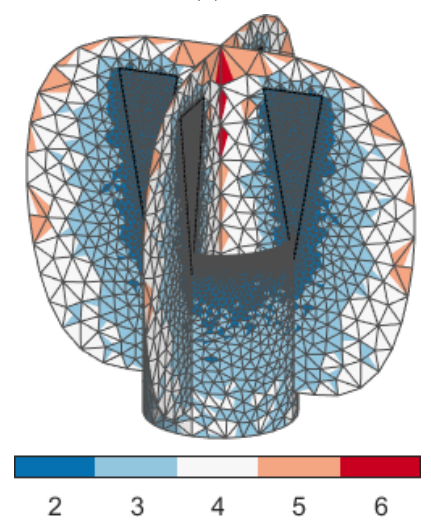

(e)

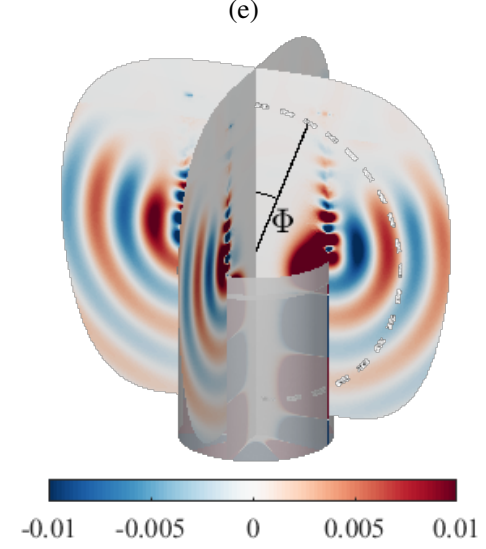

Figure 5: Acoustic radiation from a straight circular semi-infinite duct, under hot-jet exhaust flow conditions (a) Mean flow Mach number $M_{0}$ and LEE-LPE interface (solid lines), (b) Baseline mesh and order distribution from 2 to 6 used for the adaptive LEE-LPE solution with $E_{T}=1 \%$ at $k_{0} r_{\mathrm{d}}=8.5$ and (c) corresponding solution (real part of pressure) for the mode $(m, n)=(5,1)$, (d) Refined mesh with order distribution and (e) corresponding solution obtained for the mode $(m, n)=(5,1)$.

Clearly, the first two points need to be addressed on a case-by-case basis and some preliminary tests might be necessary to achieve the right balance between accuracy and efficiency.

For this test case, a conically shaped coupling interface is defined, shown by solid lines in Figure 5(a). It starts inside the duct, namely at $z=z_{d}-0.3$, and extends up to $z=z_{d}+2.1$, with the same spreading angle $\beta$ as the shear layer profile, so as to closely follow the growth of the shear layer thickness. At this interface, the conditions described in Section 3 are imposed to adequately couple the two variational formulations.

The incoming acoustic wave is the duct mode $(m, n)=(5,1)$ with the angular frequency $\omega=2892 \mathrm{rad} / \mathrm{s}$. The shortest acoustic wavelength is $0.56 \mathrm{~m}$, and is equal to the largest vorticity wavelength. In this study, the computational domain is discretised with two meshes, namely a baseline mesh and a refined mesh. The baseline grid is shown in Figure 5(b). The size of the mesh elements ranges from $0.25 \mathrm{~m}$ in the duct to $0.4 \mathrm{~m}$ at the outer boundary. The baseline mesh is slightly refined at the duct trailing edge, with a mesh size of $0.02 \mathrm{~m}$ applied at the tip, in order to take the geometric singularity into account. The grid comprises a total of 36555 10-noded quadratic tetrahedral elements in the physical region and 1644 15-noded quadratic prism elements in the PML. The coupling interface involves 2832 6-noded quadratic triangle faces. The face order distribution obtained for a user-defined accuracy of $E_{T}=1 \%$ is also shown in Figure 5(b). The a-priori error indicator automatically adjusts the finite element basis to the mean flow and the element size distribution, taking also into account the mesh curvature (see [22]). In the LEE region, the typical order is $p_{\mathrm{FEM}}=3$, except at the duct tip, where the minimal order $p_{\mathrm{FEM}}=2$ is used. In the high-Mach number region $\left(M_{0_{\mathrm{d}}}=0.5\right)$, the typical order extends from $p_{\mathrm{FEM}}=3$ to $p_{\mathrm{FEM}}=6$, thus accounting for the acoustic wavelength 
(a)

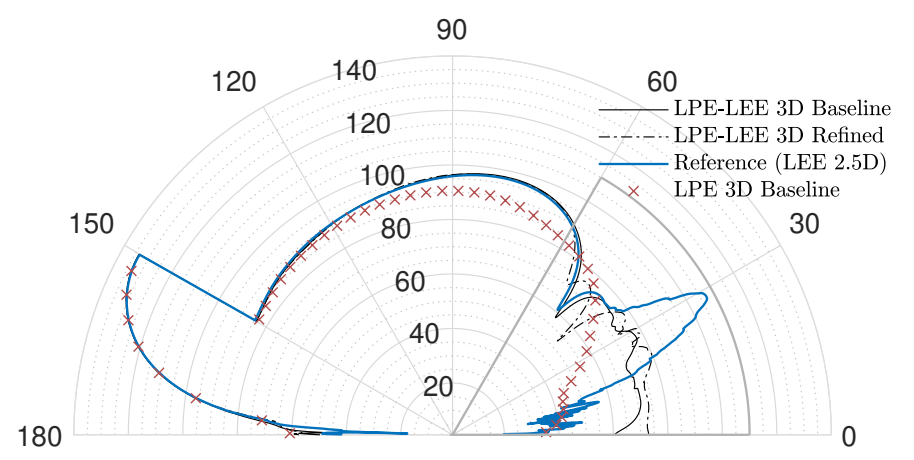

(b)

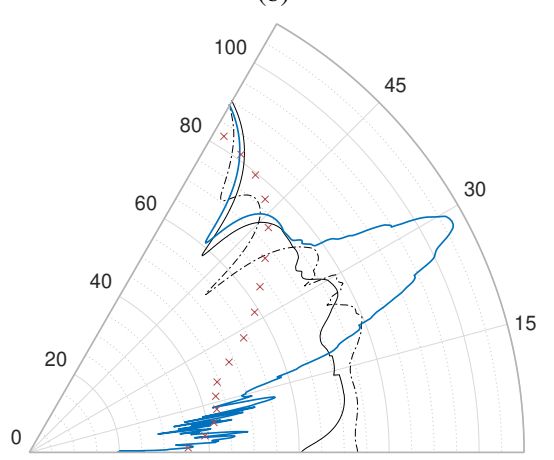

Figure 6: (a) Sound Pressure Level (in dB) obtained with the different models against the observer angle $\Phi \in\left[0^{\circ}, 180^{\circ}\right]$ along the control circle, for the mode $(5,1)$ with jet flow condition $M_{0_{\mathrm{d}}}=0.7641$ and $M_{0_{\infty}}=0$ at $\omega=2892 \mathrm{rad} / \mathrm{s}$. (b) Zoom in the lower directivity angles, in the vicinity of the vortex sheet $\Phi \in\left[0^{\circ}, 60^{\circ}\right]$.

shortening induced by the background flow. In the far-field region, where the mesh is coarsened for an improved efficiency, the order varies from $p_{\mathrm{FEM}}=3$ to $p_{\mathrm{FEM}}=5$. The refined mesh is designed in the same way as the baseline mesh, except in the shear layer region where a strong mesh refinement is applied, with $h=0.05 \mathrm{~m}$ in domain $\Omega_{\mathrm{LEE}}$. The refined mesh, colored by the face orders distributed from 2 to 6 , is presented in Figure 5(d). Note that the minimal order $p_{\text {FEM }}=2$ is used in the shear layer region.

Figures 5(c) and 5(e) present the numerical solutions (real part of pressure) obtained from the $p$-adaptive hybrid model using the baseline and refined meshes respectively. The acoustic mode propagates in the duct, is refracted through the vortex sheet and radiates to the far field. A small-amplitude spurious fluctuation is also visible at the downstream end of the LEE region, but this does not have a significant influence on the directivity, see below.

At the duct tip, the shear-layer is responsible for the generation of the hydrodynamic Kelvin-Helmholtz instability, which develops as a vorticity shedding along the duct wake and decays after a finite distance, as the thickness of the shear layer increases. The development and the attenuation of the vorticity shedding in the wake are, as expected, much more noticeable on the refined mesh solution in Figure 5(e). However, qualitatively, the radiation patterns in the far field is not altered noticeably by the instability waves.

More quantitative results are provided by comparing the numerical solutions obtained from the baseline and the refined grids on a 2 m-radius circle centered on the point $\left(0,0, l_{\mathrm{d}}\right)^{T}$. The circle is indicated by dashed lines in Figures 5(c) and 5(e). The sound pressure levels along this circle are shown in Figure 6 as a function of the angle $\Phi$ measured from the $z$-axis pointing away from the duct (see Figure 5(c)). As a reference solution, an axisymmetric (2.5D) solution of the LEE (see [6]) is also provided using a very high fixed order $p_{\text {FEM }}=8$. The two hybrid EPE 3D solutions are overall in good agreement with this reference solution, the main features of the radiated sound field are present. The pressure trace inside the $\operatorname{duct}\left(\Phi=\left[150^{\circ}, 180^{\circ}\right]\right)$ is closely matching. A slight difference in magnitude is however observed along the main directivity lobe found at $\Phi \approx 75^{\circ}$. In particular, in Fig. 6(b), at $\Phi=60^{\circ}$, the baseline mesh solution yields a typical amplification of about $1 \mathrm{~dB}$ when compared to the reference and the refined mesh solutions, which are found in close agreement. Larger differences are also noticeable for lower directivity angles, i.e. in the region of the shear layer located at $\Phi=30^{\circ}$, see Figure $6(\mathrm{~b})$. This is due to the presence of the Kelvin-Helmholtz instability whose amplitude varies on the different meshes. Another important remark is that no pressure jumps are visible when crossing the coupling interface, which indicates that the continuity of pressure is properly enforced. An LPE scalar solution without LEE patch is also computed on the baseline mesh as a reference. While it accurately predicts the solution inside the duct, it is unable to capture the main directivity lobe. Differences of 5 to $10 \mathrm{~dB}$ with respect to the $2.5 \mathrm{D}$ LEE reference solution are found in this region $\left(\Phi \approx 75^{\circ}\right)$, which illustrates that the LPE model alone is not a reliable approach for modeling noise radiation through heated jet flows.

Table 1 compares the performance of the different 3D methods in terms of number of degrees of freedom (DoFs), memory footprint and solving time. The coupled LPE-LEE adaptive method applied to the baseline mesh involves a total of 658082 DoFs with condensation of the internal bubble shape functions. Solving the corresponding linear 


\begin{tabular}{c|c|c|c|c} 
Method & Mesh & Number of DoFs * & Factorization Memory (Mb) & Solving time (sec) \\
\hline LPE only & Baseline & 318840 & 12481 & 56 \\
LPE-LEE & Baseline & 658082 & 36310 & 127 \\
LPE-LEE & Refined & 2657916 & 295173 & 1566 \\
LEE only & Baseline & 1594200 & $272631^{\dagger}$ & N.A.
\end{tabular}

Table 1: Comparison of the computational costs of the different 3D models as obtained from the MUMPS linear solver, for the sound radiation from a straight circular semi-infinite duct under hot-jet exhaust flow conditions, at $k_{0} r_{\mathrm{d}}=8.5\left(^{*}\right.$ with condensation of internal bubble DoFs, ${ }^{\dagger}$ estimated memory).

\section{Fan noise radiation through jet exhaust flow}

The proposed method is now applied to predict the propagation of fan noise through the dual-stream jet flow exhausting from a model-scale turbofan engine. This is an important application, as in modern high-bypass ratio engines, the fan noise that radiates through the exhaust nozzle is considered one of the loudest sound sources [31]. The geometry and mean flow data were generated in the framework of the European project TURNEX [32], and have been investigated in several studies [5,33]. The so-called short cowl nozzle geometry is analyzed here, under static flow conditions.

\subsection{Model preparation}

The mean flow computation was performed solving the Reynolds-Averaged Navier-Stokes (RANS) equations with the $k-\epsilon$ turbulence model [5]. In Figure 7(a), the Mach number on the original CFD mesh is shown. The flow is injected through the bypass duct $\left(M_{\mathrm{fan}}=0.35, c_{\text {fan }}=349.6 \mathrm{~m} / \mathrm{s}, \rho_{\mathrm{fan}}=1.22 \mathrm{~kg} / \mathrm{m}^{3}\right)$, while the core heated jet is considered at rest $\left(M_{\text {turb }}=0, c_{\text {turb }}=530 \mathrm{~m} / \mathrm{s}, \rho_{\text {turb }}=0.48 \mathrm{~kg} / \mathrm{m}^{3}\right)$.

The mean flow vorticity obtained from the RANS, defined as $\left|\omega_{0}\right|=\left|\nabla \times \boldsymbol{u}_{0}\right|$, is given in Figure 7(b). Notable flow hear exists in the boundary layers, nozzle exhaust areas as well as in the jet mixing regions.

This mean flow field needs to be mapped onto the (typically coarser) acoustic mesh. Previous studies have highlighted the importance of an appropriate mean flow description to ensure an efficient aeroacoustic propagation [34]. In particular, the thin boundary layers obtained from RANS which have a marginal impact on the acoustic propagation, and are typically not resolved when mapped onto the acoustic mesh, need to be removed. To decide which CFD points to keep and which to disregard, a measure of the normal distance to the wall is required. In this work, the wall-distance method proposed by Tucker [35] is used. In this approach, wall-normal distances are inferred from the solution of an in-homogeneous Poisson equation with Dirichlet boundary conditions applied on the wall surfaces. The latter is carried out using conventional linear finite elements on the original RANS mesh, and the resulting sparse linear system is solved using an iterative conjugate gradient procedure. The wall-normal distances $d_{\mathrm{n}}$ obtained using this strategy are shown in Figure 7(c). It is worth emphasizing that, despite the geometry curvature, the wall-normal distance remains smooth throughout the domain. A wall-distance threshold of $d_{\mathrm{n}}<6 \mathrm{~mm}$ is applied for the boundary layer truncation (BLT). When the location where an approximation is desired falls within this region, it is extrapolated from the CFD 
(a) Mach number $M_{0}$

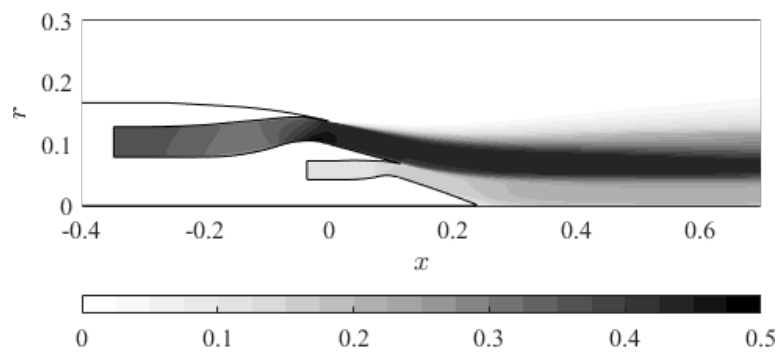

(c) Distance to wall $d_{\mathrm{d}}$

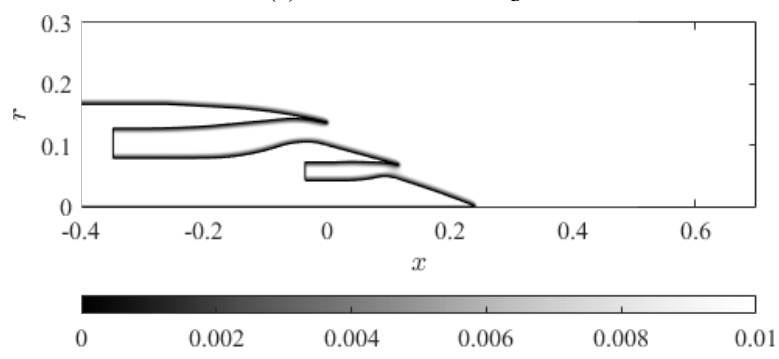

(b) Vorticity magnitude $\left|\omega_{0}\right|=\left|\nabla \times \boldsymbol{u}_{0}\right|$

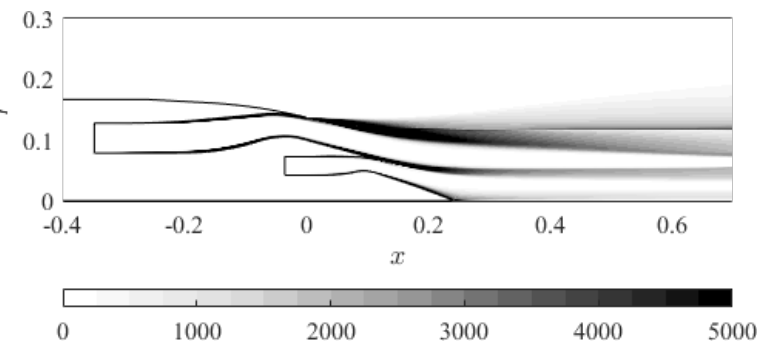

(d) Vorticity (after BLT) + LEE-LPE Coupling interface

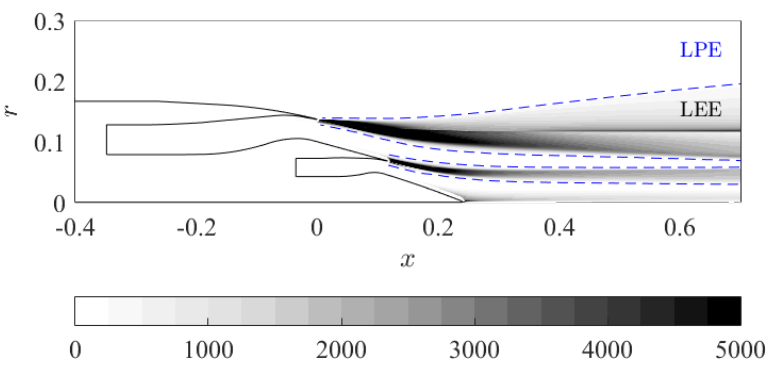

Figure 7: Turnex short cowl test case: (a) mean flow Mach number and (b) vorticity fields obtained from RANS for static approach condition (courtesy of Brian Tester, ISVR), (c) distance to wall obtained by solving an inhomogeneous Poisson problem (see [35]), (d) vorticity magnitude after boundary layer truncation, also showing the LEE-LPE coupling interface in blue obtained from the isoline $\left|\omega_{0}\right|=200 \mathrm{~s}^{-1}$.

data outside of the boundary-layer region. The resulting mean flow vorticity magnitude, after application of the BLT, is shown in Figure 7(d). No flow vorticity is visible in the near-wall regions, which indicates that the boundary layers have been efficiently removed.

Once the mean flow representation is finalized, the different regions of the proposed hybrid propagation model may be assigned. The separation is applied automatically, based on an isoline of the vorticity magnitude, namely $\left|\omega_{0}\right|=200 \mathrm{~s}^{-1}$ (see Figure 7(d)). The two strong shear flow regions, where $\left|\omega_{0}\right|>200 \mathrm{~s}^{-1}$, encompassing the bypass and the core jet mixing regions, are modeled using the LEE, whereas the rest of the domain, namely, the in-duct and far-field propagation regions, are modeled using the LPE. The value $\left|\omega_{0}\right|>200 \mathrm{~s}^{-1}$ is problem-specific and another value would have to be chosen for a different test case.

\subsection{Numerical results}

In the acoustic simulations, the computational domain extends from $x=-0.36 \mathrm{~m}$ to $x=0.54 \mathrm{~m}$ and is surrounded by a cylindrical PML of radius $0.3 \mathrm{~m}$ and of width $0.2 \mathrm{~m}$. For this study, two acoustic meshes, referred to as fine and coarse grids, are created using Gmsh [29], with a respective characteristic element size of $h=4 \mathrm{~cm}$ and $h=6 \mathrm{~cm}$. A cross section of the two meshes is provided in Fig. 8(a) and 8(b). In both cases, in order to maintain an accurate geometry and mean flow representation, a curvilinear quadratic mesh is generated, yielding 10-noded tetrahedrons in the physical domain and 15-noded prisms in the PML. A mesh refinement $h_{\min }=h / 5$ is also applied at the tips of the exhaust and core ducts, to account for the presence of a geometric singularity. The coarse mesh comprises 25565 elements and 50776 nodes, while the fine mesh involves 62649 elements and 125380 nodes.

The mean flow obtained after the boundary layer truncation is mapped from the RANS structured grid onto the acoustic quadratic mesh nodes, using bilinear interpolation. Figure 9(a) and 9(b) respectively present the interpolated Mach number $M_{0}$ and speed of sound $c_{0}$ obtained on the coarse acoustic mesh $(h=6 \mathrm{~cm})$. The mapped mean flow remains smooth across the shear layers. The interface $\Gamma$ separating the LPE from the two LEE domains is materialized by thick solid lines. It can be verified that the regions of strong flow gradients are enclosed within the bypass and core LEE sub-regions. 
(a) Fine mesh $h=4 \mathrm{~cm}$

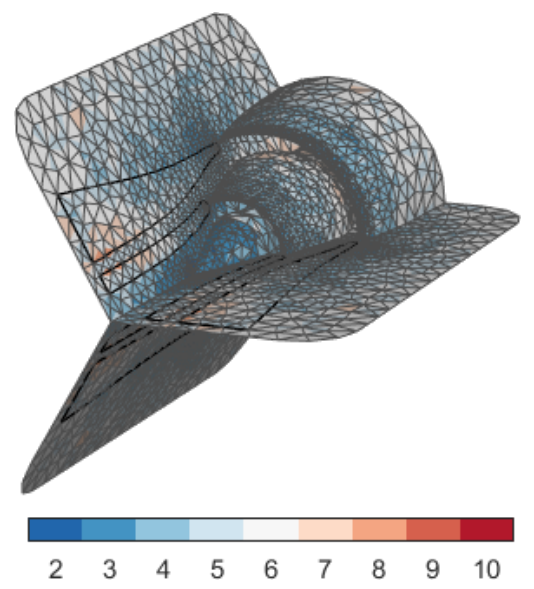

(b) Coarse mesh $h=6 \mathrm{~cm}$

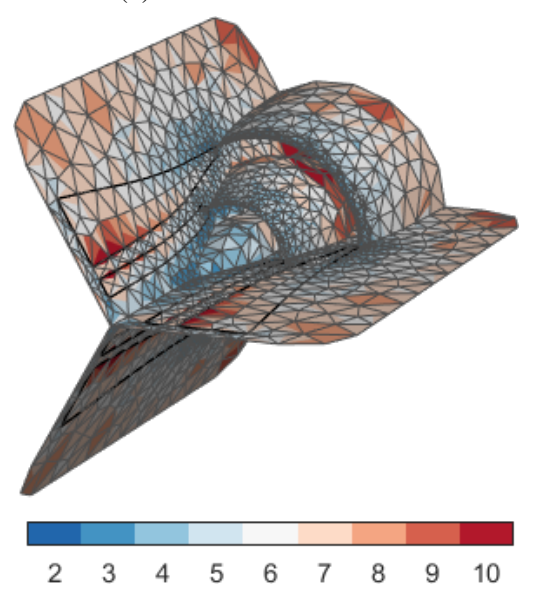

Figure 8: Meshes and element order distribution for the short-cowl nozzle TURNEX test case under static approach condition at $f=7497 \mathrm{~Hz}$ (target accuracy $E_{T}=1 \%$ ). The LEE-LPE coupling interfaces in the bypass and core mixing regions are indicated with straight lines.

The frequency is set to $f=7497 \mathrm{~Hz}$ which corresponds to a Helmholtz number $k r_{\text {fan }}$ of 26.5 , following the definition provided in [9]:

$$
k r_{\mathrm{fan}}=\frac{2 \pi f r_{\mathrm{fan}}}{c_{\mathrm{fan}}\left(1-M_{\mathrm{fan}}\right)} .
$$

Figure 8 also presents the face order distribution, as obtained from the a priori error indicator with a target accuracy of $E_{T}=1 \%$. The adaptive approach automatically adjusts the polynomial order based on the frequency, the local grid size and the local mean flow properties. The typical order is $p_{\mathrm{FEM}}=5-6$ for the refined mesh and $p_{\mathrm{FEM}}=7-8$ for the coarse mesh. While the order is naturally elevated in higher flow speed regions, lower orders are used in the mesh refinement area but also in the heated core jet, where the speed of sound increases, as visible in Fig 9(b).

To model the noise contribution from the fan, a set of incoming annular acoustic duct modes $(m, n)$ are generated in the bypass cross-section at $x=-0.348 \mathrm{~m}$, using an active version of the PML. Three duct modes are considered in this analysis, the plane wave mode $(m, n)=(0,1)$, and two well cut-on modes, namely $(m, n)=(4,1)$ and $(m, n)=(9,1)$. All individual modes are prescribed at the inlet with a unit incident modal intensity. Figure 10 presents the real part of the pressure obtained with the hybrid adaptive model on the coarse mesh, for the three individual incident duct modes. The solutions are qualitatively satisfactory, the pressure distribution is continuous throughout the domain and the presence of the physical interface is not apparent. The duct modes, generated in the bypass duct, are refracted through the shear layer and radiate to the far field. The mode $(9,1)$ radiates with a higher angle and is characterized by one main directivity lobe, whereas the mode $(4,1)$ exhibits multiple secondary lobes. The mode $(0,1)$ on the other hand radiates at lower angles and thereby, a significant portion of the acoustic energy remains confined in the jet flow. This makes the plane wave mode propagation particularly challenging to simulate, as the vorticity and acoustic perturbations are expected to interact over a larger distance, downstream of the core and bypass mixing regions.

As discussed in Section 4.2, the proposed adaptive scheme adjusts the polynomial order so as to only resolve the range of acoustic length scales. As a result, no K-H instabilities are visible in 10. By contrast, previous analysis performed on the same case (using fixed-order, over-resolved solutions) have consistently reported the presence of spatially growing hydrodynamic perturbations in the bypass shear-layer [36].

In order to examine the numerical solutions in more detail, Figure 11 presents the sound pressure levels obtained along a circular control line of radius $0.3 \mathrm{~m}$, centered at the tip of the bypass duct. As a reference for the quantitative verification of the three-dimensional LEE-LPE hybrid model, a full LEE axisymmetric (2.5D) solution is computed, using a mesh size $h=2 \mathrm{~cm}$ and a low target accuracy $E_{T}=10^{-2} \%$. The sound pressure levels obtained with the proposed hybrid model on the fine mesh $(h=4 \mathrm{~cm})$ are in close agreement with the reference solution. The main directivity lobes and associated peak pressure levels are well predicted. The solution obtained using the coarse mesh reveals larger differences with respect to the reference solution. This is thought to be related to the mean flow and 
(a) Mach number $M_{0}$

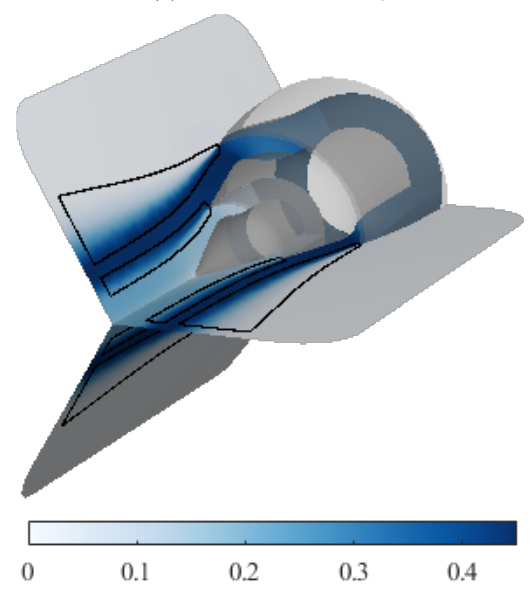

(b) Speed of sound $c_{0}, \mathrm{~m} / \mathrm{s}$

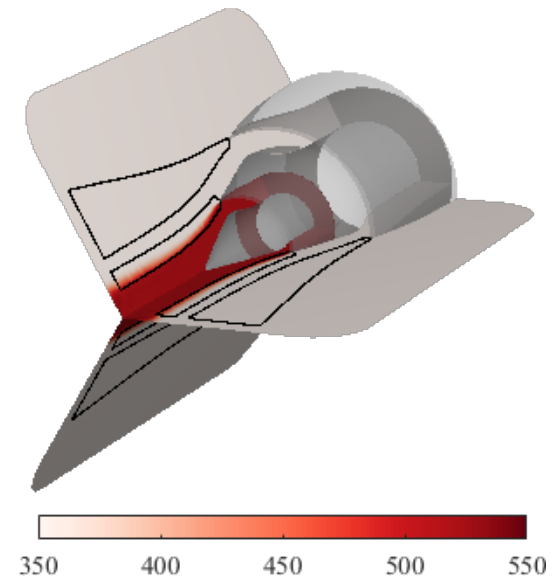

Figure 9: Interpolated mean flow on the mesh with $h=6 \mathrm{~cm}$ for the short-cowl nozzle TURNEX test case under static approach condition. The LEE-LPE coupling interfaces in the bypass and core mixing regions are indicated by black solid lines.

\begin{tabular}{c|c|c|c|c|c} 
Method & Mesh & $\left(p_{\min }, p_{\max }\right)$ & Number of DoFs & Factorization Memory $(\mathrm{Mb})$ & Solving time (min) \\
\hline LPE-LEE 3D & Fine & $(2,8)$ & 2888295 & $431612^{\dagger}$ & $68(\mathrm{OOC})$ \\
LPE-LEE 3D & Coarse & $(2,10)$ & 1888986 & 267834 & 32 \\
LEE only 3D & Coarse & $(2,10)$ & 5618602 & $3022626^{\dagger}$ & N.A.
\end{tabular}

Table 2: Comparison of the computational costs of the different 3D models as obtained from the MUMPS linear solver, for the TURNEX shortcowl use case under hot-jet exhaust flow condition, at $7497 \mathrm{~Hz}\left({ }^{*}\right.$ with condensation of internal bubble DoFs, ${ }^{\dagger}$ estimated memory, OCC designates out-of-core computation).

geometric representation, which are more crudely represented on the coarse mesh. Furthermore, significant differences are noticeable at low directivity angles $\left(\Phi<30^{\circ}\right)$ in the region of the shear layer, particularly for the mode $(9,0)$. This is due to the presence of a localized K-H instability in the $2.5 \mathrm{D}$ reference solution, which is not apparent in the 3D adaptive model. Overall, the proposed LEE-LPE adaptive model yields satisfactory results, the main features of the sound radiated from the exhaust are well captured, even on the coarse mesh.

The computational cost of the different 3D numerical models is now investigated, see Table 2. The coarse mesh model with typical order $p_{\text {FEM }}=7-8$, involves 1888986 DoFs, of which roughly 50\% (872 276 DoFs) are used for the discretization of the shear flow regions in domain $\Omega_{\mathrm{LEE}}$. It was solved in-core by the MUMPS library in 32 minutes on 16 threads and required 267 GB of physical memory.

The simulation on the refined mesh on the other hand, with typical element order $p_{\text {FEM }}=5-6$ required up to 2888295 DoFs, and was solved out-of-core in 68 minutes. This is in agreement with conclusions from previous studies which have indicated that, at fixed accuracy, higher-order elements lead to lower memory requirements, especially when static condensation is applied $[19,8,22]$. Whenever possible, coarse meshes should therefore be advocated, as they allow to better exploit the accuracy of the high-order approximation basis. A three-dimensional full LEE model was also assembled on the coarse mesh, as a reference. According to MUMPS estimates, the in-core factorization of the resulting system matrix would have required approximately 3 TB of RAM. In this particular example, the proposed LPE-LEE hybrid model therefore allowed to reduce the memory footprint of the simulation by more than an order of magnitude, without appreciably compromising the accuracy of the solution.

\section{Conclusions}

An efficient strategy has been proposed for the modeling of acoustic propagation through vortical flows. In this approach, regions of high-shear flows, where vorticity and entropy effects are expected to play an important role, are modeled using the accurate, albeit expensive, linearised Euler equations, while the rest of the domain is modeled using 
(a) Mode $(0,1)$

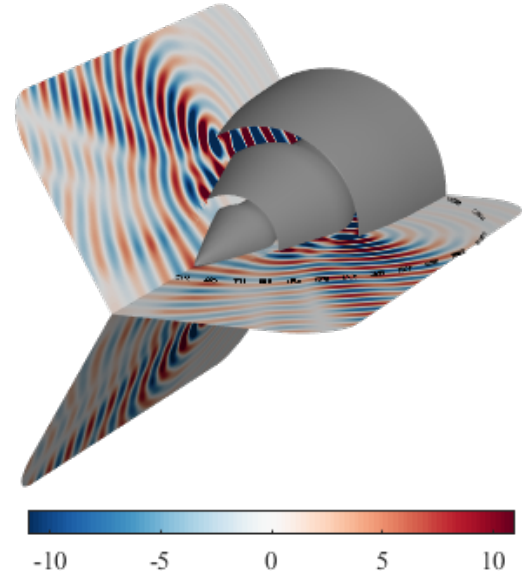

(b) Mode $(4,1)$

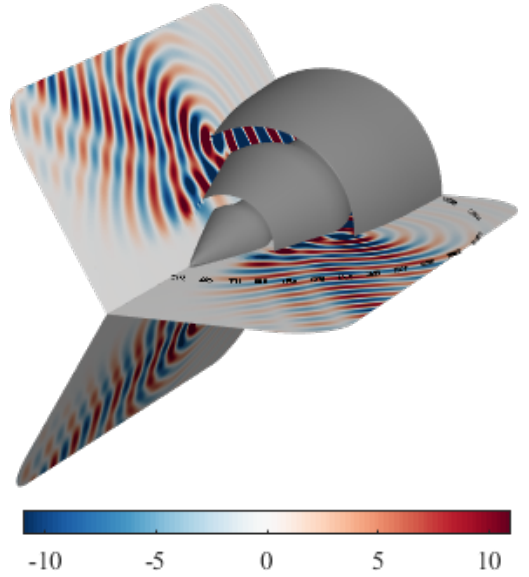

(c) Mode $(9,1)$

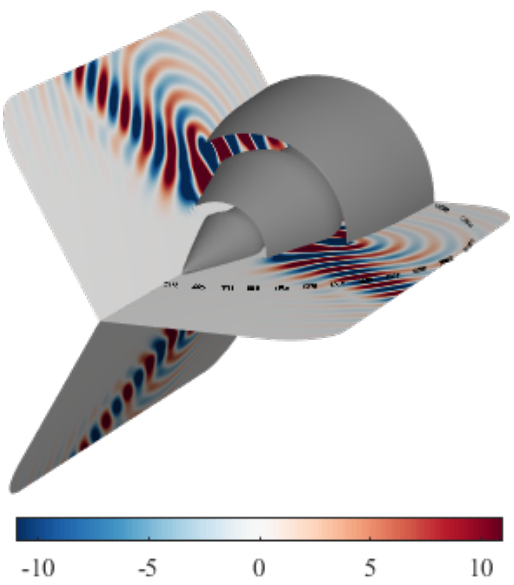

Figure 10: Real part of pressure obtained with the adaptive hybrid LEE-LPE model on the mesh $h=6 \mathrm{~cm}$, at $7497 \mathrm{~Hz}$ for various incident modes enforced in the bypass duct. The control circle used for the directivity measurements is also shown (dashed line).

(a) Mode $(0,1)$

90

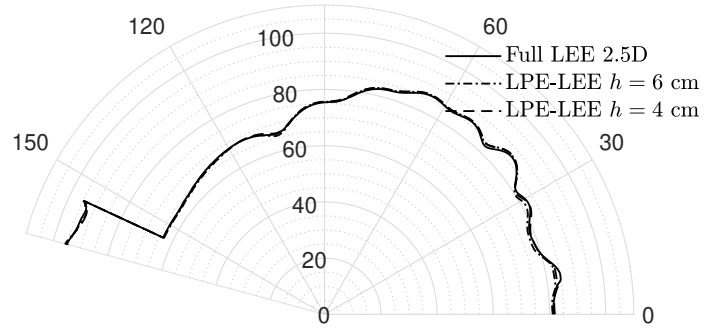

(b) Mode $(9,1)$

90

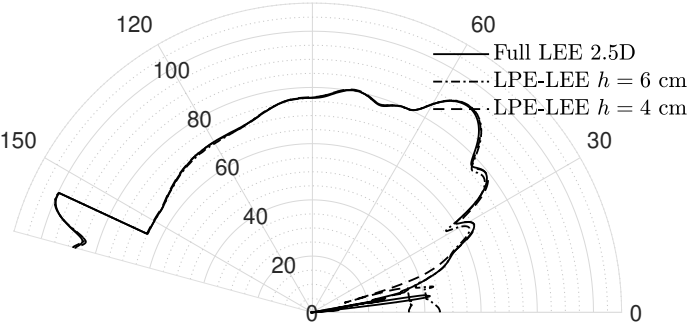

Figure 11: Sound Pressure Level (in dB) against the position angle $\Phi \in\left[0^{\circ}, 165^{\circ}\right]$ along the control circle, for the short-cowl nozzle TURNEX exhaust radiation at $f=7497 \mathrm{~Hz}$.

the scalar linearised potential equation. Transmission conditions have been introduced to allow for an efficient coupling at the physical interface between the two propagation models. To further enhance the computational efficiency, the resulting coupled problem is discretized using a high-order adaptive Finite Element strategy, which adjusts the polynomial order across the mesh automatically at each frequency, accounting for the local grid and mean flow properties. Several numerical examples are provided which illustrate the performance of the method, including a lab-scale turbofan exhaust application under realistic static flow conditions.

Other applications of this hybrid approach could be considered. For instance, LEE regions could be used to represent sound propagation through boundary layers, while using the LPE for the propagation of sound in the rest of the domain.

A configuration that has not been considered in the present paper is the case where the interface corresponds to an outflow boundary for the LEE region and vorticity or entropy waves are present in the LEE region. In this case it is likely that the interface conditions formulated here will result in spurious reflections from the interface since it does not act as a non-reflecting boundary condition for these waves. This effect remains to be assessed in more detail.

Another aspect that has not been addressed in the present paper is situations where the LEE-LPE interface extends into the Perfectly Matched Layer. In this case one would have to derive modified coupling conditions specifically for the portion of the interface inside the PML since the governing equations have been modified to implement the PML. 


\section{Acknowledgments}

The author gratefully acknowledge the European Commission (EC) for its support through the FP7-PEOPLEITN-2011 FLOWAIRS project (Silent Air Flows in transport, buildings and power generation, www.flowairs.eu, grant 289352) and the CRANE project (Community and Ramp Aircraft NoisE, www.crane-eid.eu, grant 606844). The authors would also like to thank Dr. Brian Tester for providing the TURNEX exhaust geometry and mean flow data and acknowledge Dr. Michael Williamschen for suggesting the boundary layer truncation approach.

\section{Appendix A. Flux matrices of linearised Euler equations}

The flux matrices in equation (1) are defined as follows:

$$
\begin{aligned}
& \boldsymbol{A}=\left[\begin{array}{ccccc}
0 & 1 & 0 & 0 & 0 \\
-u_{0}^{2} & 2 u_{0} & 0 & 0 & \rho_{0} c_{0}^{2} / p_{\mathrm{c} 0} \\
-u_{0} v_{0} & v_{0} & u_{0} & 0 & 0 \\
-u_{0} w_{0} & w_{0} & 0 & u_{0} & 0 \\
-p_{\mathrm{c} 0} u_{0} / \rho_{0} & p_{\mathrm{c} 0} / \rho_{0} & 0 & 0 & u_{0}
\end{array}\right] \\
& \boldsymbol{B}=\left[\begin{array}{ccccc}
0 & 0 & 1 & 0 & 0 \\
-u_{0} v_{0} & v_{0} & u_{0} & 0 & 0 \\
-v_{0}^{2} & 0 & 2 v_{0} & 0 & \rho_{0} c_{0}^{2} / p_{\mathrm{c} 0} \\
-v_{0} w_{0} & 0 & w_{0} & v_{0} & 0 \\
-p_{\mathrm{c} 0} v_{0} / \rho_{0} & 0 & p_{\mathrm{c} 0} / \rho_{0} & 0 & v_{0}
\end{array}\right] \\
& \boldsymbol{C}=\left[\begin{array}{ccccc}
0 & 0 & 0 & 1 & 0 \\
-u_{0} w_{0} & w_{0} & 0 & u_{0} & 0 \\
-v_{0} w_{0} & 0 & w_{0} & v_{0} & 0 \\
-w_{0}^{2} & 0 & 0 & 2 w_{0} & \rho_{0} c_{0}^{2} / p_{\mathrm{c} 0} \\
-p_{\mathrm{c} 0} w_{0} / \rho_{0} & 0 & 0 & p_{\mathrm{c} 0} / \rho_{0} & w_{0}
\end{array}\right]
\end{aligned}
$$

\section{References}

[1] H. Bériot, G. Gabard, K. Hamiche, M. Williamschen, High-order unstructured methods for computational aero-acoustics, in: Progress in simulation, control and reduction of ventilation noise, Publisher: von Karman Institute for Fluid Dynamics, 2015.

[2] H. L. Atkins, C.-W. Shu, Quadrature-free implementation of discontinuous Galerkin method for hyperbolic equations, AIAA Journal 36 (5) (1998) 775-782.

[3] C. Bailly, D. Juvé, Numerical Solution of Acoustic Propagation Problems Using Linearized Euler Equations, AIAA Journal 38 (1) (2000) 22-29.

[4] P. P. Rao, P. J. Morris, Use of Finite Element Methods in Frequency Domain Aeroacoustics, AIAA Journal 44 (7) (2006) 1643-1652.

[5] Y. Özyörük, B. J. Tester, Application of Frequency-Domain Linearized Euler Solutions to the Prediction of Aft Fan Tones and Comparison with Experimental Measurements on Model Scale Turbofan Exhaust Nozzles, Journal of Sound and Vibration 330 (16) (2011) 3846-3858.

[6] K. Hamiche, G. Gabard, H. Bériot, A High-Order Finite Element Method for the Linearised Euler Equations, Acta Acustica United With Acustica 102 (5) (2016) 813-823.

[7] R. J. Astley, R. Sugimoto, P. Mustafi, Computational Aero-Acoustics for Fan Duct Propagation and Radiation. Current Status and Application to Turbofan Liner Optimisation, Journal of Sound and Vibration 330 (26) (2011) 3832-3845.

[8] H. Bériot, A. Prinn, G. Gabard, Efficient Implementation of High-Order Finite Elements for Helmholtz Problems, International Journal for Numerical Methods in Engineering 106 (3) (2016) 213-240.

[9] G. Gabard, H. Bériot, A. Prinn, K. Kucukcoskun, Adaptive, high-order finite-element method for convected acoustics, AIAA Journal 56 (8) (2018) 3179-3191.

[10] M. E. Goldstein, An Exact Form of Lilley's Equation with a Velocity Quadrupole / Temperature Dipole Source Term, Journal of Fluid Mechanics 443 (2001) 231-236.

425 [11] R. Leveque, Finite volume methods for hyperbolic problems, Cambridge University Press, 2002.

[12] R. L. Higdon, Initial-Boundary Value Problems for Linear Hyperbolic Systems, SIAM Review 28 (2) (1986) 177-217.

[13] A. Bermúdez, L. Hervella-Nieto, A. Prieto, R. Rodríguez, An Optimal Perfectly Matched Layer with Unbounded Absorbing Function for Time-Harmonic Acoustic Scattering Problems, Journal of Computational Physics 223 (2) (2007) 469-488.

[14] E. Bécache, A.-S. B.-B. Dhia, G. Legendre, Perfectly Matched Layers for the Convected Helmholtz Equation, SIAM Journal on Numerical Analysis 42 (1) (2004) 409-433. 
[15] M. Gaborit, O. Dazel, P. Göransson, G. Gabard, Coupling of finite element and plane waves discontinuous Galerkin methods for timeharmonic problems, International Journal for Numerical Methods in Engineering 116 (7) (2018) 487-503.

[16] P. Šolín, K. Segeth, I. Doležel, Higher-Order Finite Element Methods, CRC Press, Boca Raton, FL, USA, 2004.

[17] G. E. Karniadakis, S. Sherwin, Spectral/hp Element Methods for Computational Fluid Dynamics, Oxford University Press, 2013.

[18] P. E. J. Vos, S. J. Sherwin, R. M. Kirby, From $h$ to $p$ efficiently: Implementing finite and spectral/hp element methods to achieve optimal performance for low- and high-order discretisations, Journal of Computational Physics 229 (13) (2010) 5161-5181.

[19] A. Lieu, G. Gabard, H. Bériot, A comparison of high-order polynomial and wave-based methods for Helmholtz problems, Journal of Computational Physics 321 (2016) 105-125.

[20] A. El Kacimi, O. Laghrouche, M. Mohamed, J. Trevelyan, Bernstein-Bézier based finite elements for efficient solution of short wave problems, Computer Methods in Applied Mechanics and Engineering 343 (2019) 166-185.

[21] H. Bériot, G. Gabard, E. Perrey-Debain, Analysis of high-order finite elements for convected wave propagation, International Journal for Numerical Methods in Engineering 96 (11) (2013) 665-688.

[22] H. Bériot, G. Gabard, Anisotropic adaptivity of the p-FEM for time-harmonic acoustic wave propagation, Journal of Computational Physics 378 (2019) 234-256.

445 [23] M. Goldstein, Aeroacoustics, New York, McGraw-Hill International Book Co., 1976.

[24] G. G. Vilenski, S. W. Rienstra, Numerical study of acoustic modes in ducted shear flow, Journal of Sound and Vibration 307 (3-5) (2007) 610-626.

[25] A. Michalke, Survey on jet instability theory, Progress in Aerospace Sciences 21 (1984) 159-199.

[26] B. Tester, G. Gabard, Y. Özyörük, Influence of mean flow gradients on fan exhaust noise predictions, in: 14th AIAA/CEAS Aeroacoustics Conference (29th AIAA Aeroacoustics Conference), AIAA Paper 2008-2825, 2008

[27] A. Agarwal, P. J. Morris, R. Mani, The Calculation of Sound Propagation in Nonuniform Flows: Suppression of Instability Waves, AIAA Journal 42 (1) (2004) 80-88.

[28] A. Iob, R. Arina, C. Schipani, Frequency-Domain Linearized Euler Model for Turbomachinery Noise Radiation Through Engine Exhaust, AIAA Journal 48 (4) (2010) 848-858.

[29] C. Geuzaine, J.-F. Remacle, Gmsh: A 3-D finite element mesh generator with built-in pre-and post-processing facilities, International Journal for Numerical Methods in Engineering 79 (11) (2009) 1309-1331.

[30] P. R. Amestoy, I. S. Duff, J. Koster, J.-Y. L'Excellent, A fully asynchronous multifrontal solver using distributed dynamic scheduling, SIAM Journal on Matrix Analysis and Applications 23 (1) (2001) 15-41.

[31] R. Astley, R. Sugimoto, I. Achunche, M. Kewin, P. Mustafi, E. Deane, Reprint of: A review of CAA for fan duct propagation and radiation, with application to liner optimisation, Procedia IUTAM 1 (2010) 143-152.

[32] B. J. Tester, F. Arnold, S. Caro, S. Lidoine, TURNEX: Turbomachinery Noise Radiation Through the Engine Exhaust. Publishable Final Activity Report, Tech. rep., E.U. Project 516079 (2008).

[33] A. Iob, R. D. R. Rinaldi, R. Arina, A Frequency-Domain Linearized Euler Model for Noise Radiation, in: Aeronautics and Astronautics, ISBN: 978-953-307-473-3, IntechOpen, 2011

[34] M. Williamschen, G. Gabard, H. Bériot, Impact of the mean flow representation on DGM simulations of flow acoustics, in: 22nd AIAA/CEAS Aeroacoustics Conference, AIAA Paper 2016-2974, 2016.

[35] P. Tucker, Assessment of geometric multilevel convergence robustness and a wall distance method for flows with multiple internal boundaries, Applied Mathematical Modelling 22 (4-5) (1998) 293-311

[36] K. Hamiche, A high-order finite element model for acoustic propagation, Ph.D. thesis, University of Southampton (2016). 


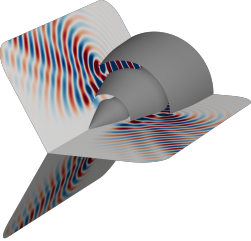

